\title{
Nowcasting Register Labour Force Participation Rates in Municipal Districts Using Survey Data
}

\author{
Jan van den Brakel ${ }^{1}$ and John Michiels ${ }^{1}$
}

\begin{abstract}
In the Netherlands, very precise and detailed statistical information on labour force participation is derived from registers. A drawback of this data source is that it is not timely since definitive versions typically become available with a delay of two years. More timely information on labour force participation can be derived from the Labour Force Survey (LFS). Quarterly figures, for example, become available six weeks after the calendar quarter. A wellknown drawback of this data source is the uncertainty due to sampling error. In this article, a nowcast method is proposed to produce preliminary but timely nowcasts for the register labour force participation on a quarterly frequency at the level of municipalities and neighbourhoods, using the data from the LFS. As a first step, small area estimates for quarterly municipal figures on labour force participation are obtained using the LFS data and the unitlevel modelling approach of Battese, Harter and Fuller (1988). Subsequently, time series of these small area estimates at the municipal level are combined with time series on register labour force participation in a bivariate structural time series model in order to nowcast the register labour force participation at the level of municipalities and neighbourhoods.
\end{abstract}

Key words: Small area estimation; unit-level model; survey sampling; register-based statistics; data integration.

\section{Introduction}

Official statistics on the labour force are traditionally obtained by using probability sampling in combination with design-based or model-assisted inference procedures (see Cochran (1977) or Särndal et al. (1992) for an introduction). For national statistical institutes (NSIs) this is a widely accepted approach, since it allows to draw valid inferences about finite populations based on relatively small samples, where it is understood that the size of the sample is small with respect to the population size. In addition, the uncertainty of observing a small sample instead of the entire target population can be quantified through variance calculation. Because of the continuing need of NSIs to reduce costs and response burden, alternative data sources are being explored. An important example is the use of data sources available outside statistical agencies, such as register data from the tax service or local population registers (e.g., see Wallgren and Wallgren 2007, chap. 1; Hand 2018). More recently, also other data sources that are not

\footnotetext{
${ }^{1}$ Statistics Netherlands (CBS), PO Box 4481, Heerlen 6401 CZ, the Netherlands. Emails: JBRL@cbs.nl and jjm.michiels@cbs.nl

Acknowledgments: The views expressed in this article are those of the authors and do not necessarily reflect the policies of Statistics Netherlands. The authors are grateful to the Associate Editor and six anonymous referees for careful reading and commenting on two former drafts of this manuscript. Their comments proved to be very helpful to improve this article.
} 
directly related to a statistical or an administrative purpose are considered in the production of official statistics, so-called big data (see e.g., Daas and Puts 2014; Pfeffermann et al. 2015).

Administrative data used in the application described in this article provide an almost complete enumeration of the target population and are therefore useful in the production of detailed statistics. This can be done by using administrative data as a primary data source for producing statistics (Wallgren and Wallgren 2007, chap. 1) or as auxiliary information in small area estimation procedures (Rao and Molina 2015, chap. 5, 7). Other benefits sometimes granted to administrative data and big data are their timeliness. This, however, varies between applications. If administrative data or big data sets become available at a higher frequency than repeated surveys, than their timeliness can be exploited in nowcasting methods and small area estimation methods. The auxiliary information from these more timely data sets can be used to produce more accurate estimates for the sample survey in real time even in instances when the statistics from a big data source become available but the sample data are not yet collected and processed (Vosen and Schmidt 2011; Choi and Varian 2012; Giannone et al. 2008).

Typical examples of big data sources that are available at a high frequency are Google trends, statistics derived from social media platforms, sensor data, mobile phone data, data obtained from GPS trackers, and scanner data.

Administrative data are not necessarily timely. Income tax registers, for example, come in the Netherlands with a delay of two years. In this case, their additional value comes from the fact that they cover the target population almost entirely. In such cases, more timely statistics can be produced with survey samples, with the obvious drawback that sufficient precise estimates are only available at high regional levels. If a repeated survey is conducted to produce more timely statistics than administrative sources can, nowcasting methods can be used to combine both data sources and produce preliminary estimates at a detailed level. An example of this is considered in this article, where the Dutch Labour Force Survey (LFS) and tax registers in the Netherlands provide information about the employed labour force.

For the purpose of producing statistics on the labour force participation rate at a low regional level, the traditional approach of survey sampling and design-based estimators was, until recently, the preferred method in the Netherlands. Until 2015, municipal estimates were produced annually by means of direct general regression estimation (GREG) (see, for example Särndal et al. 1992, chap. 6), for municipalities with at least 30,000 inhabitants. To improve annual municipal estimates, a model-based small area estimation method (SAE) has been implemented. Small area estimation refers to a class of model-based estimation procedures that explicitly rely on a statistical model to improve the precision of sample estimates. Mainstream SAE methods are based on cross-sectional multilevel models, which improve the precision of domain estimates with sample information observed in other domains observed in the same reference period. Official statistics, however, are generally produced repeatedly in time. For such situations, multivariate time series models are appropriate since they improve sample estimates with sample information observed in previous reference periods as well as information observed in other domains. A comprehensive overview of the literature on SAE is provided by Rao and Molina (2015). For a more compact, but nevertheless complete overview of the SAE literature, see Pfeffermann (2002, 2013). 
Statistics Netherlands uses a unit level model (Battese et al. 1988), for the estimation of labour status at the level of municipalities at an annual frequency. See Boonstra et al. (2011) and CBS Statline, (2017) for details. Still, this latter approach is not ideal for statistics at a very low regional level, such as districts within municipalities, or other very small subdomains. Small area estimators for very small domains reduce to purely synthetic estimators, which may be severely biased.

An alternative approach is to use register data to produce statistics on the employed labour force. If tax data are available at the individual level about persons with paid employment and persons who are self-employed, detailed statistics on the labour force participation rate can be produced for small domains. The register alternative can deliver labour force statistics at a regional level within municipalities, although it may fall short when there is a need for timely statistics on the labour force participation rate.

For very detailed and timely regional statistics (or other statistics for small domains) each of the latter two techniques are therefore in many cases insufficient. They may, however, be combined to produce more accurate results using nowcasting methods. Many official statistics are produced repeatedly. Using information from the past to make more precise estimates for the last period is possible in a time series approach. This idea has been pursued by many authors dating back to research by Blight and Scott (1973). Moreover, auxiliary time series that correlate with the target series may be used to improve the accuracy of the target series. Other key references to authors that apply time series methods to survey data are Rao and Yu (1994), Pfeffermann and Burck (1990), Harvey and Chung (2000) and Pfeffermann and Tiller (2006).

The Netherlands is divided into 12 provinces and contains about 400 municipalities. Municipalities are further divided into neighborhoods, about 12,000 in total. The purpose of this article is to use more timely quarterly survey data on labour force participation at the municipal level to nowcast less timely register statistics at the more detailed level of neighbourhoods. To this end, the bivariate state space model developed by Van den Brakel et al. (2017) is further extended in this article. Quarterly time series of labour force participation rates for approximately 12,000 neighbourhoods have been derived from register sources. Alongside these register series quarterly survey time series of labour force participation rates at the municipal level are derived for the time period 2003-2014 using small area predictions obtained using a cross-sectional unit level model. The approach applied in this article is to use the register time series up to 2013 and the LFS time series up to 2014 in order to nowcast the register statistics for the periods 2012-2014. These predictions are then compared with the final register statistics.

The novelty of this article can be found in the following extensions. A timely time series obtained with a repeated survey is used as an auxiliary series in a state-space model to nowcast a very detailed target series that comes from a register with a time lag of two years. A crosssectional unit level model is applied to the survey data as a first step, which is used to produce auxiliary series for the state-space model. The state-space model accounts for the uncertainty in the auxiliary series, as well as the autocorrelation, which arise from the rotating panel design of the LFS. Finally, the model is applied to the COVID-19 pandemic, for the purpose of evaluating the nowcast procedure directly after a turning point induced by the COVID-19 crisis.

In Section 2, the survey design of the labour force survey and its regional estimation procedure is described. Also described is the construction of regional statistics on the 
employed labour force using register data from the tax service. In Section 3, structural time series models are proposed for the survey and register time series on labour force participation rates over the time period 2003-2014. Both univariate and bivariate models are considered and the results obtained by these models are presented in Section 4. The article concludes with a discussion in Section 5.

\section{Official Statistics About the Dutch Labour Force}

\subsection{Labour Force Survey}

\subsubsection{Survey Design}

The target population of the Dutch LFS is defined as persons aged 15 to 75 years residing in the Netherlands and not living in an institutional household. Since 2000, the Dutch LFS is based on a rotating panel design, in which respondents are interviewed five times at quarterly intervals. Each month a sample of addresses is selected through a stratified twostage cluster design. Strata are formed by geographic regions. Municipalities are considered as primary sampling units and addresses as secondary sampling units. All households residing at an address, up to a maximum of three, are included in the sample. About 9,000 new respondents are interviewed monthly in the first wave of the panel design. Until 2010, data collection in the first wave was based on computer-assisted personal interviewing (CAPI) only. From 2010 until 2012, data collection in the first wave changed to a mixed-mode data collection approach of computer-assisted telephone interviewing (CATI) if a non-secret landline number was available, and CAPI for the remaining households. Since 2012, data collection in the first wave is based on a sequential mixed-mode design that starts with computer-assisted web interviewing (CAWI). After three reminders, non-respondents are approached by means of CATI if a non-secret landline number is available, and by CAPI for the remaining households. In the four subsequent waves of the panel, data are collected by means of CATI, since the start of the panel in 2000. During these re-interviews, a reduced questionnaire is completed to establish changes in the labour market position of the household members aged 15 years and over. When a household member cannot be contacted, proxy interviewing is allowed by members of the same household in each wave. Labour force participation is established by asking respondents about (not) having a paid job.

The aforementioned redesigns in 2010 and 2012 resulted in systematic effects in the LFS estimates. The series observed before 2012 are adjusted to the level of the sequential mixed mode design observed since 2012. See Van den Brakel and Krieg (2015) for more details on how to quantify and correct for these so-called discontinuities in the Dutch LFS.

\subsubsection{Estimation Procedures for Official Labour Force Figures}

LFS data are used to publish official statistics about the labour force on a monthly, quarterly and annual frequency. Inference methods of the LFS are based on a mixture of design-based and model-based techniques. Monthly figures about the unemployed, employed and total labour force are based on a multivariate structural time series modelling approach. This method improves the precision compared to direct estimates by 
using sample information from preceding periods, accounts for rotation group bias and autocorrelation induced by the rotating panel design of the LFS. Monthly figures are published at the national level and as a breakdown in six domains based on a crossclassification of age and gender (see Van den Brakel and Krieg (2015) for details).

Quarterly figures at the national level are based on the GREG estimator. The weighting procedure contains the monthly estimates from the time series model to force consistency between monthly and quarterly figures about the labour force. Several registrations provide additional auxiliary information that is used in this weighting procedure. These auxiliary variables include registered paid employment (source: Polis administration), which is a strong predictor for labour force participation.

Regional quarterly and annual information on the labour force is provided by the LFS in official statistics using small area techniques for the estimation of labour force indicators at the municipal and province level, since sample sizes are too small to use direct estimators at this level. The backbone of this modelling approach is an hierarchical Bayesian version of the Battese-Harter-Fuller unit-level model (Battese et al. 1988; Datta and Ghosh 1991). Various labour force indicators are presented for a number of subpopulations (according to sex, age groups, educational level and ethnicity). The most detailed regional indicators (at the municipal level) appear on a yearly basis, within two months after the reference year has ended.

Quarterly sample sizes of the LFS amount to approximately 85,000 persons, distributed across just over 400 municipalities. Municipal sample sizes vary between zero for the smallest municipalities and over 3,000 for the capital, Amsterdam. In Figure 1 a Pareto graph visualises the distribution of the sample over the municipalities for the first quarter of 2014. For the purposes of this publication, the small area estimates of all municipalities have been estimated on a quarterly basis using the aforementioned Battese-Harter-Fuller unit-level model. For a given period $t$, let $y_{i, d}^{L F S}$ denote a binary indicator for labour force participation and $x_{i, d}$ a vector of covariates for sample unit $i$ and municipality $d$. The linear

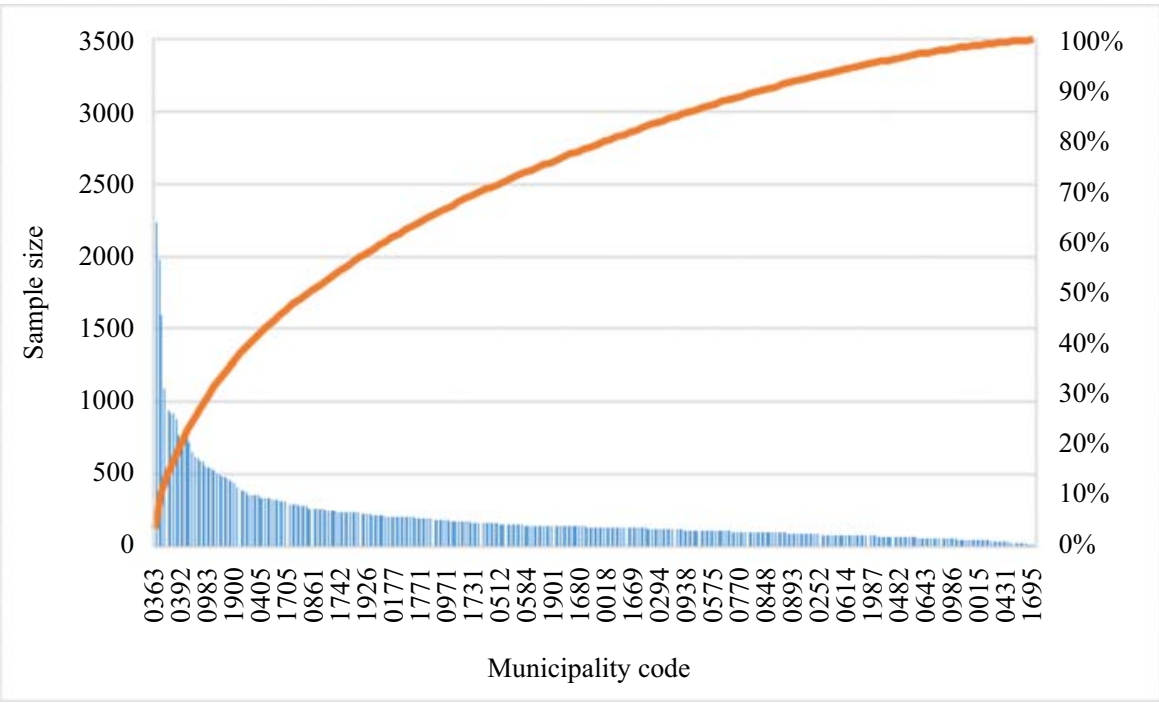

Fig. 1. Pareto graph of municipal sample sizes in the Dutch LFS for the first quarter of 2014. 
unit level model is used as an approximation for the observed variables, that is, $y_{i, d}^{L F S}=\beta^{\prime} x_{i, d}+v_{d}+\varepsilon_{i, d}$, with $\beta$ a vector of regression coefficients, $v_{d} \cong N\left(0, \sigma_{v}^{2}\right)$ an i.i.d. random domain effect and $\varepsilon_{i d} \cong N\left(0, \sigma_{\varepsilon}^{2}\right)$ i.i.d. random errors. Let $\bar{y}_{d}^{L F S}$ denote the sample mean of the labour force participation rate of municipality $d$. Based on this model, empirical best linear unbiased predictions for the domains are obtained by

$$
\begin{gathered}
y_{d}^{L F S}=\hat{\gamma}_{d}\left(\bar{y}_{d}^{L F S}+\hat{\beta}^{\prime}\left(\bar{X}_{d}-\bar{x}_{d}\right)\right)+\left(1-\hat{\gamma}_{d}\right) \hat{\beta}^{\prime} \bar{X}_{d}, \\
\hat{\gamma}_{d}=\frac{\hat{\sigma}_{v}^{2}}{\hat{\sigma}_{v}^{2}+\hat{\sigma}_{\varepsilon}^{2} / n_{d}}, \hat{\beta}=\left(X^{\prime} \hat{\Sigma}^{-1} X\right)^{-1} X^{\prime} \hat{\Sigma}^{-1} y .
\end{gathered}
$$

Here, $n_{d}$ is the number of observations in municipality $d, n=\sum_{d=1}^{D} n_{d}$ is the number of observations in a particular period $t, D$ is the total number of municipalities, $\bar{x}_{d}$ is a vector with sample means of municipality $d$ and $\bar{X}_{d}$ is a vector with the corresponding population means, $X$ is the full matrix with covariates of the $n$ units included in the sample, $y$ is the $n$ vector with observations of the $n$ units included in the sample, and $\hat{\Sigma}_{t}=\operatorname{cov}\left(y_{t}\right)=\hat{\sigma}_{\varepsilon, t}^{2} I_{n_{t}}+\hat{\sigma}_{v, t}^{2} \oplus_{d=1}^{D} J_{n_{t, d}}$, where $I_{n}$ is the identity matrix of order $n$ and $\oplus_{d=1}^{D} J_{n_{t, d}}$ is the block diagonal matrix of order $n \times n$ with $J_{n_{d}}$ the $n_{d} \times n_{d}$ block diagonal elements with each element equal to 1 . Note that the empirical best linear unbiased prediction estimator defined in Equation (1) can also be expressed as $y_{d}^{L F S}=\hat{\beta}^{\prime} \bar{X}_{d}+\hat{\gamma}_{d}\left(\bar{y}_{d}^{L F S}-\hat{\beta}^{\prime} \bar{x}_{d}\right)$. The advantage of Equation (1) is that it has the interpretation that the estimator is the weighted average of the survey regression estimator $\hat{\gamma}_{d}\left(\bar{y}_{d}^{L F S}-\hat{\beta}^{\prime} \bar{x}_{d}\right)$ and the regression synthetic estimator $\hat{\beta}^{\prime} \bar{X}_{d}$, see Rao and Molina (2015, subsec. 7.2). The model used in Equation (1) is:

$$
\begin{aligned}
& \text { EmployedReg } \times[\text { Gender }+\operatorname{Age}(3)+\text { Ethinicity(3) }+ \text { Bordermunicipality(2) }] \\
& + \text { Gender } \times \text { Age }(3)+\operatorname{Age}(5)+\text { Ethnicity(7) }+ \text { EmploymentOffice(5) } \\
& + \text { HHType }(3)+\text { Wage }(6)+\text { WaveNr }
\end{aligned}
$$

where the variables are explained in Appendix (Subsection 6.1). The demographic auxiliary variables in the model are available from the Municipal Basic Administration (MBA). This is a register of all people residing in the Netherlands and contains background variables such as age, sex, nationality, and marital status. Since Dutch citizens are required by law to report changes in their demographics to their municipalities, this register provides a very accurate list of the Dutch population (Bakker 2012). EmployedReg and EmploymentOffice are available from registers of the Employee Insurance Agency and can be linked with the MBA through a personal identifier. Each person residing in the Netherlands has a unique personal identifier that is available in most registrations.

Equation (1) is expressed as an hierarchical Bayesian model with a flat prior on $\beta, \sigma_{v}^{2}$ and $\sigma_{\varepsilon}^{2}$ and fitted using the R-package hbsae, (Boonstra 2012). Small area predictions and their standard errors are obtained from the posterior mean and posterior variance of $y_{d}^{L F S}$.

Applying a linear model directly to binary data or percentages might appear rigid at first sight but similar linear models are used to motivate the general regression estimator that is generally used in survey sampling to estimate sample means or totals of binary or categorical variables. Boonstra et al. (2007) conducted a large simulation study for the Dutch municipal labour force figures, in which it was found that logistic unit-level models do not improve upon normal linear models. Furthermore, the quantities of interest are area means. Interest is not 
focused in predictions for individual units, but always aggregate such predictions to the area level. This makes it more reasonable to use normal linear models. Finally, prediction for nonlinear models is computationally more cumbersome than for linear models and often not worthwhile the effort. See, for example, Bijlsma et al. (2020) for another application in which logistic models do not outperform the normal linear model. Selection and evaluation of this model is described in detail in Boonstra et al. (2007, 2008, 2011). Other examples where the area level model is applied to untransformed estimated percentages in the context of SAE are Datta et al. (1999), You et al. (2003), and Arima et al. (2017). Early references on the use of the logistic-normal model for SAE are MacGibbon and Tomberlin (1989) and Malec et al. (1997). See Hobza and Morales (2016), Hobza et al. (2018), and Marino et al. (2019) for more recent literature on alternative ways of obtaining small area predictions for binary data under a logistic mixed model. In this article, the linear Equation (1) is applied, since this is the model that is used by Statistics Netherlands for the production of official quarterly LFS figures.

Instead of cross-sectional small area estimation models, time series small area estimation models can be used as an alternative. Rao and Yu (1994) extended the crosssectional area level model by Fay and Herriot (1979) with an AR(1) component to borrow strength over time and space. Harvey and Chung (2000) proposed a time series model for the LFS in the United Kingdom extended with a series of claimant counts. Pfeffermann and Burck (1990) and Pfeffermann and Tiller (2006) developed multivariate structural state space models to borrow strength over time and space. Boonstra and Van den Brakel (2019) emphasise that structural time series models can be considered as time series extensions of the area level model, which both can be expressed as state space models or time series multilevel models, resulting in similar estimation results.

\subsection{Register Data for Low Regional Statistics on the Employed Labour Force}

The register data contain information about the sources of income of persons on a monthly basis, including income from paid employment, self-employment and social benefits. These register data are based on data collected by the tax service (De Belastingdienst) and the Employee Insurance Agency. Self-employed taxpayers may opt for a delayed tax declaration and the final tax assessment may take several years. As a result, data collection is significantly slower than with the LFS. Approximately nine months after the reference year has ended a preliminary data set is produced and the final one is produced the year after. These data sets can be combined with other register data that contain individual information about demographic and regional variables among other variables.

In Figure 2, time series are presented on quarterly small domain estimates of labour force participation rates from the LFS and quarterly figures on labour force participation rates derived from the tax register. The municipal domain estimates are based on the Battese-Harter-Fuller unit-level model. The time series contain quarterly data over the period 2003-2014 for three municipalities: Amsterdam, the largest Dutch municipality (811,000 inhabitants, January 2014); Heerlen, a medium-size municipality in the southeast corner of the Netherlands (88,000 inhabitants, January 2014) and Haren, a relatively small municipality in the north (14,000 inhabitants, January 2014).

For the three municipalities, it follows that the results for labour force participation rates are very close, which is not trivial. In many situations, survey data and related register data 


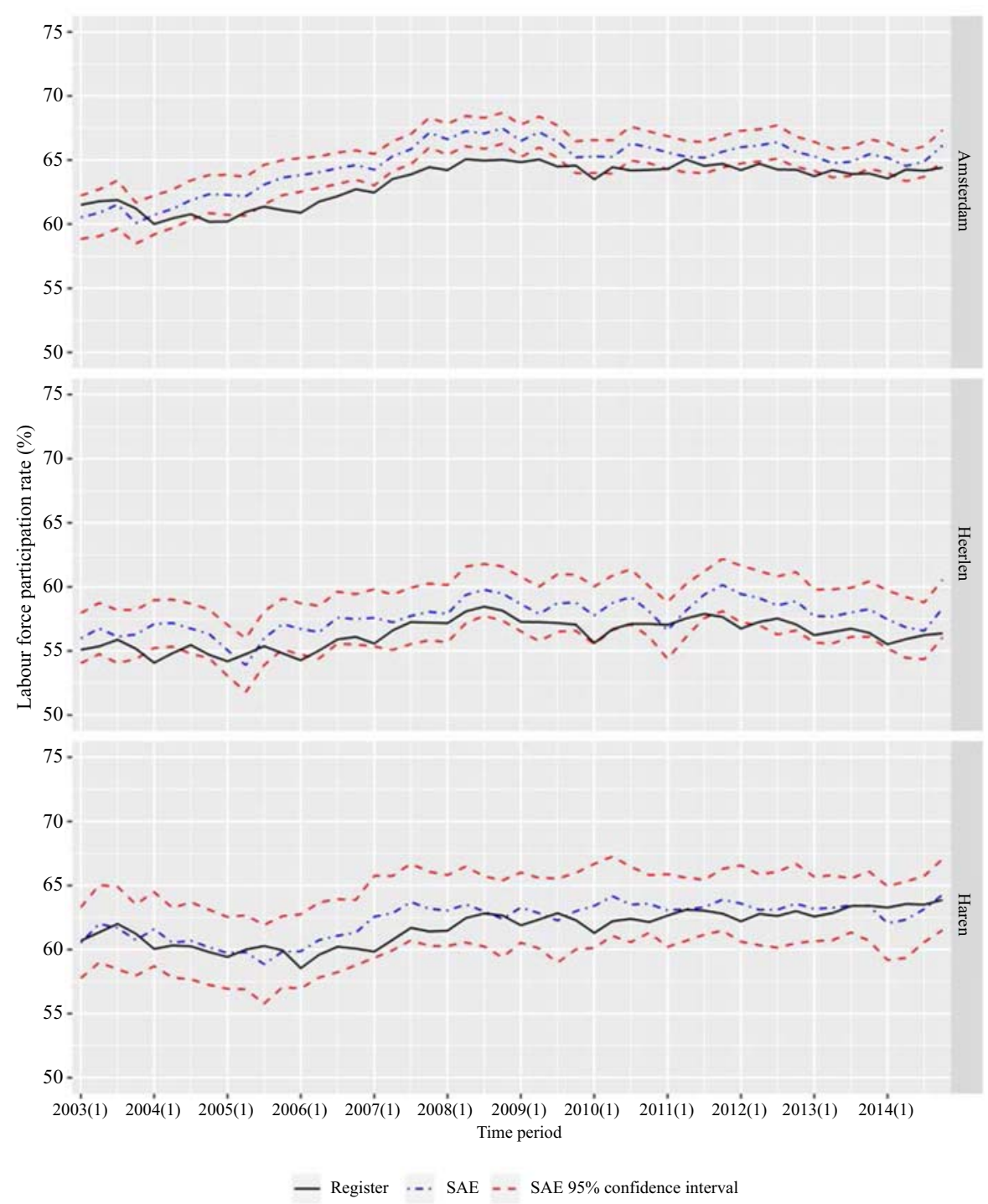

Fig. 2. LFS and register labour force participation rates for a large, medium-sized and small municipality respectively, quarterly data 2003-2014 with a 95\% confidence interval for the LFS SAE statistics.

follow a similar evolution but generally at different levels. Although a small area estimation approach is used to estimate the labour force participation rate with the LFS data, the amount of uncertainty increases inversely proportional to the sample size of the domains.

\subsection{Accuracy of Survey and Register Statistics Concerning Regional Labour Force Statistics}

The accuracy of statistics is usually measured in terms of bias and variance. We here distinguish between selection bias, bias introduced by the estimation procedure and measurement bias. Variance constitutes an important part of the uncertainty of LFS labour 
force indicators, especially for small domains (municipal regions) in which the sample sizes are limited. To overcome this problem, small area estimators are used for the calculation of labour force participation rates at the municipal level. These estimators are not unbiased, but in the presence of strong auxiliary information using models that meet their underlying assumptions, this bias might be limited and the variance reduction dominates. As a result, the mean squared error of these estimators is smaller than the variance of traditional direct (GREG) estimators.

Another contributing factor to the uncertainty of survey statistics is selection bias. In theory, this bias is zero under complete response. In practice, selection bias arises due to undercoverage of the sampling frame, the inability to reach the target population with the implemented field work strategies and data collection mode successfully, and selective nonresponse. In register data, there is no uncertainty due to a sampling design. However there still is uncertainty about labour force participation rate statistics due to incomplete data collection in the preliminary tax datasets. The uncertainty stemming from temporary nonresponse is partially removed by imputing the missing records for the self-employed in a deterministic procedure (by checking the existence of provisional assessments and companies' registry membership). Imputing for missing values may and does lead to biased estimates. Apart from temporary nonresponse, there are also groups that do not need to declare their income (e.g., some groups of household workers) and workers that do not declare their taxable income although they should (informal labour market).

In survey sample statistics, the measurement bias depends on the extent to which conceptual variables to be measured are operationalised correctly in the questionnaire. However, the mode of data collection and other contributing factors (such as the quality of the interviewers, and errors introduced elsewhere in the production process) are relevant.

Register data are not without measurement bias. In addition, taxpayers have to complete a questionnaire (their tax declaration) and usually do this without the help of an interviewer. The difficult nature of the tax form may lead to measurement bias. Furthermore, there may be underreporting of income sources. However, it is assumed in this application that these errors can be ignored.

\section{Time Series Modelling of Register and Survey Indicators of Labour Force Participation}

In this section, univariate and bivariate structural time series models are developed for the quarterly statistics on labour force participation rates obtained with the LFS and the tax register. With a structural time series model, a series is decomposed into a trend component, a seasonal component, other cyclic components, a regression component and an irregular component. For each component, a stochastic model is assumed. This allows the trend, seasonal, and cyclic component, but also the regression coefficients, to be time dependent. If necessary, ARMA components can be added to capture the autocorrelation in the series beyond these structural components. See Harvey (1989) or Durbin and Koopman (2012) for details about structural time series modelling.

The question addressed in this article is whether time series from an auxiliary source at an aggregate level (e.g., municipalities) with more timely data than the main time series, but hampered with sampling error, can be used to make more precise first predictions (nowcasts) 
for the measurements in the main series at a more detailed regional level (neighbourhoods). The auxiliary series is considered useful if the combination of both series leads to smaller mean square errors for the nowcasted estimates than in the case of a univariate modelling approach of the main series. The main time series is based on register data on labour force participation rates at the level of municipalities and underlying neighbourhoods. The auxiliary series consists of timely estimates of labour force participation rates at the level of municipalities or more aggregated regional units obtained from the LFS. The question is tackled by developing a bivariate structural time series model for the register series (for a municipality or neighbourhoods within a municipality) and the LFS series (for the corresponding municipality or a higher level regional unit) and modelling the correlation between the disturbance terms of the different components of the structural time series for both models.

The model selection starts by building appropriate univariate time series models for the labour force participation rates obtained with the LFS and the tax register data. This forms the input for building an optimal bivariate model. The performance of this bivariate model will be compared with predictions based on the univariate model for labour force participation rates. Results are presented for three municipalities: a large municipality, Amsterdam, together with a mediumsized and a small municipality, respectively: Heerlen and Haren. In this fashion, the effect of larger standard errors for estimates in smaller municipalities can be included in the analysis. In a further stage of the analysis register, labour force participation rates for neighbourhoods will also be related to the corresponding survey indicator at the municipal level.

\subsection{Univariate Models for Register and Survey Statistics on Labour Participation}

Let $y_{t, d}^{R}$, denote the register measurements of labour force participation rate for period $t$ of municipality $d$ and $y_{t, d}^{L F S}$ the survey estimates for labour force participation rate for period $t$ of municipality $d$ based on the LFS and estimated with the HB model defined in Equation (1). For both time series, the following structural time series model is proposed:

$$
y_{t, d}^{x}=L_{t, d}^{x}+S_{t, d}^{x}+E_{t, d}^{x} \text { with } x \in\{R, L F S\},
$$

with $L_{t}^{x}$ and $S_{t}^{x}$ an appropriate stochastic model for the trend and the seasonal component and $E_{t}^{x}$ the remaining unexplained variation.

For both time series, the smooth trend model appears to be the most appropriate model to capture both trend and cyclic components. The smooth trend model is defined as (Durbin and Koopman, 2012, Subsubsec. 3.2.1):

$$
\begin{gathered}
L_{t, d}^{x}=L_{t-1, d}^{x}+R_{t-1, d}^{x}, \\
R_{t, d}^{x}=R_{t-1, d}^{x}+\eta_{t, d}^{x}, \eta_{t, d}^{x} \cong N\left(0, \sigma_{\eta, d}^{x^{2}}\right), \operatorname{Cov}\left(\eta_{t, d}^{x}, \eta_{t^{\prime}, d}^{x}\right)=0 \text { if } t \neq t^{\prime} .
\end{gathered}
$$

In Equation (4) $L_{t, d}^{x}$ is the level of the trend, which is defined as the level of the previous period plus a change, $R_{t-1, d}^{x}$ which can be interpreted as a slope parameter. The slope on its turn is modelled as a random walk. As a result, the trend can gradually change over time. Therefore, Equation (4) models the low frequency variation of the series and has the flexibility to model the trend and long-term economic cycles. The flexibility of the trend component is determined by the value of the variance of the slope disturbance terms, that is, $\sigma_{\eta, d}^{x^{2}}$. If $\sigma_{\eta, d}^{x^{2}}=0$, then Equation (4) defines a straight line, that is, $L_{t, d}^{x}=L_{0, d}^{x}+t R_{0, d}^{x}$, 
with $L_{0, d}^{x}$ the intercept which defines the level of the trend at the start of the series and $R_{0, d}^{x}$ a time-invariant slope.

Alternative trend models are the local linear trend model and the local level model. The local linear trend model is obtained if an additional random component is added to $L_{t, d}^{x}$ in Equation (4). In that case, the first line of Equation (4) equals $L_{t, d}^{x}=L_{t-1, d}^{x}+R_{t-1, d}^{x}+\zeta_{t, d}^{x}$, with $\zeta_{t, d}^{x}$ a level disturbance term that is normally and independently distributed, that is $\zeta_{t, d}^{x} \cong N\left(0, \sigma_{\zeta, d}^{x^{2}}\right), \operatorname{Cov}\left(\zeta_{t, d}^{x}, \zeta_{t^{\prime}, d}^{x}\right)=0$ if $t \neq t^{\star}$.

The local level model is obtained if $L_{t, d}^{x}$ is modelled as a random walk without a slope parameter $R_{t, d}^{x}$, that is, $L_{t, d}^{x}=L_{t-1, d}^{x}+\zeta_{t, d}^{x}$. The linear trend model and the local level model generally give more volatile trend estimates compared to the smooth trend model.

A likelihood-ratio test shows that the local linear trend model for the register series does not significantly improve model fit since the log-likelihoods for a smooth trend model and the local linear trend model are almost equal. A local level model for the register series tends to overfit the data. This means that the maximum likelihood estimates for the variance of the trend disturbance terms are large, while the variance of the measurement errors tend to zero. As a result, the filtered and smoothed signals of the time series model are almost identical to the observed time series and have extremely small confidence intervals. The local level model also leads to nearly identical log-likelihoods as compared to the other two model types. Note that the likelihood of a structural time series model is based on the one-step-ahead-prediction error decomposition (Harvey 1989, subsec. 3.4). Since a model that overfit the data has low prediction power, the log-likelihood is not improved compared to the more parsimonious smooth trend model. For the survey series, the local level model and local linear trend model both tend to overfit, hence once again the smooth trend model is selected for the LFS series.

The seasonal component is modelled with a trigonometric model. For a quarterly series, this model is defined as (Durbin and Koopman, 2012, subsubsec. 3.2.2):

$$
S_{t, d}^{x}=\sum_{j=1}^{2} \gamma_{j t, d}^{x}
$$

with

$$
\begin{gathered}
\gamma_{j t, d}^{x}=\gamma_{j t-1, d}^{x} \cos \left(\lambda_{\mathrm{j}}\right)+\tilde{\gamma}_{j t-1, d}^{x} \sin \left(\lambda_{\mathrm{j}}\right)+\omega_{j t, d}^{x}, \\
\tilde{\gamma}_{j t, d}^{x}=-\gamma_{j t-1, d}^{x} \sin \left(\lambda_{\mathrm{j}}\right)+\tilde{\gamma}_{j t-1, d}^{x} \cos \left(\lambda_{\mathrm{j}}\right)+\tilde{\omega}_{j t, d}^{x},
\end{gathered}
$$

and

$$
\lambda_{\mathrm{j}}=\frac{\pi \times \mathrm{j}}{2} .
$$

For the disturbance terms it is assumed that:

$$
\begin{gathered}
\omega_{j t, d}^{x} \cong \mathrm{N}\left(0, \sigma_{\omega, d}^{x^{2}}\right), \tilde{\omega}_{j t, d}^{x} \cong \mathrm{N}\left(0, \sigma_{\omega, d}^{x^{2}}\right), \\
\operatorname{Cov}\left(\omega_{j t, d}^{x}, \omega_{j^{\prime} t^{\prime}, d}^{x}\right)=0 \text { and } \operatorname{Cov}\left(\tilde{\omega}_{j t, d}^{x}, \tilde{\omega}_{j^{\prime} t^{\prime}, d}^{x}\right)=0 \text { if } t \neq t^{\prime} \text { or } j \neq j^{\prime}, \\
\operatorname{Cov}\left(\omega_{j t, d}^{x}, \tilde{\omega}_{j^{\prime} t^{\prime}, d}^{x}\right)=0 .
\end{gathered}
$$

This means that the variance structure for all the frequency contributions is assumed to be identical with no correlation between the disturbance terms of these contributions. The 
interpretation of Equation (5) is that a quarterly seasonal pattern is modelled with a set of trigonometric terms at the seasonal frequencies $\lambda_{1}=\pi / 2$ and $\lambda_{2}=\pi$. Equation (5) shows that $\tilde{\gamma}_{1 t, d}^{x}$ contributes indirectly to the seasonal pattern via $\gamma_{1 t, d}^{x}$. Since $\sin (\pi)=0$, there are effectively three parameters, that is, $\gamma_{1 t, d}^{x}, \tilde{\gamma}_{1 t, d}^{x}$ and $\gamma_{2 t, d}^{x}$ to model the quarterly seasonal pattern. For a more detailed discussion of the trigonometric seasonal model, see Harvey (1989, subsubsec. 2.3.4).

The unexplained variation for the register series can be modelled as a white noise process, that is, $E_{t, d}^{R} \equiv \xi_{t, d}^{R} \cong N\left(0, \sigma_{\xi, d}^{R^{2}}\right)$. For the LFS series, the situation is more complicated since the unexplained variation contains the unexplained variation of the real but unknown population parameter, say $\xi_{t, d}^{L F S}$, plus the sampling error, say $e_{t, d}^{L F S}$. As a result, it follows that $E_{t, d}^{L F S}$ can be decomposed as $E_{t, d}^{L F S}=\xi_{t, d}^{L F S}+e_{t, d}^{L F S}$. The unexplained variation of the population parameter is modelled as a white-noise disturbance term, $\xi_{t, d}^{L F S} \cong N\left(0, \sigma_{\xi, d}^{L F S^{2}}\right)$. Due to the rotating panel design of the LFS, there is a large sample overlap between adjacent quarters, which results in a significant positive autocorrelation in the sampling error $e_{t, d}^{L F S}$. Furthermore, the variance of the sampling error changes over time because the sample size of the LFS change over time. Let $M S E\left(y_{t, d}^{L F S}\right)$ denote the estimated variances of the quarterly small domain predictions obtained with Battese-Harter-Fuller unit-level Equation (1). The time series model accommodates for the heteroscedasticity in the sampling error by scaling the sampling errors with the estimated mean squared errors of the quarterly small domain predictions, that is, $e_{t, d}^{L F S}=\sqrt{\operatorname{MSE}\left(y_{t, d}^{L F S}\right)} \tilde{e}_{t, d}^{L F S}$. In a next step, the Yule-Walker equations are applied to the autocovariances of the estimated sampling errors to derive an appropriate AR model for the autocorrelation in the sampling errors. It is established that an $\mathrm{AR}(1)$ model is sufficient to model the autocorrelation between the survey errors. As a result, the scaled sampling errors are modelled as $\tilde{e}_{t, d}^{L F S}=\rho_{d} \tilde{e}_{t-1, d}^{L F S}+\varsigma_{t, d}^{L F S}$, with $\rho_{d}$ the autoregressive parameter obtained from the YuleWalker equations and $\varsigma_{t, d}^{L F S} \cong N\left(0, \sigma_{s, d}^{2}\right)$. This is in line with the findings of Van den Brakel and Krieg (2015) and Boonstra and Van den Brakel (2019). Using the variance of $e_{t, d}^{L F S}$ and the coefficient for the AR(1) model derived from the micro data as a priori information in the time series models allows the identification of the following structure for the unexplained variation of the LFS series:

$$
\begin{gathered}
E_{t, d}^{L F S}=\xi_{t, d}^{L F S}+e_{t, d}^{L F S} \equiv \xi_{t, d}^{L F S}+\sqrt{M S E\left(y_{t, d}^{L F S}\right)} \tilde{e}_{t, d}^{L F S} \\
\tilde{e}_{t, d}^{L F S}=\rho_{d} \tilde{e}_{t-1, d}^{L F S}+\varsigma_{t, d}^{L F S}
\end{gathered}
$$

Note that $\operatorname{Var}\left(e_{t}^{L F S}\right)=\operatorname{MSE}\left(y_{t, d}^{L F S}\right) \sigma_{\varsigma, d}^{2} /\left(1-\rho_{d}^{2}\right)$. The variance component $\sigma_{\varsigma, d}^{2}$ is estimated via maximum likelihood (see Subsection 3.4). If $M S E\left(y_{t, d}^{L F S}\right)$, is a good approximation of the variance of $e_{t}^{L F S}$, that is, if $\operatorname{Var}\left(e_{t, d}^{L F S}\right) \approx \operatorname{MSE}\left(y_{t, d}^{L F S}\right)$, then $\sigma_{s, d}^{2} /\left(1-\rho_{d}^{2}\right)$ is expected to be close to one, and thus $\sigma_{s, d}^{2} \approx\left(1-\rho_{d}^{2}\right)$. In this way, $\sigma_{s, d}^{2}$ is in fact a scaling factor for $\operatorname{MSE}\left(y_{t, d}^{L F S}\right)$ to correct for possible bias in the estimates for $\operatorname{MSE}\left(y_{t, d}^{L F S}\right)$ that are used as a priori information in the time series model.

\subsection{Bivariate Model for Register and Survey Statistics on Labour Participation}

The univariate models for the register and LFS series can be combined in one bivariate model. This model can be used to nowcast the register series at the level of 
neighbourhoods with the more timely LFS series at the municipal level. For the bivariate model, the following structure is proposed

$$
\left(\begin{array}{c}
y_{t, d}^{R} \\
y_{t, d}^{L F S}
\end{array}\right)=\left(\begin{array}{c}
L_{t, d}^{R} \\
L_{t, d}^{L F S}
\end{array}\right)+\left(\begin{array}{c}
S_{t, d}^{R} \\
S_{t, d}^{L F S}
\end{array}\right)+\left(\begin{array}{c}
0 \\
e_{t, d}^{L F S}
\end{array}\right)+\left(\begin{array}{c}
\xi_{t, d}^{R} \\
\xi_{t, d}^{L F S}
\end{array}\right) .
$$

The models for the trend, seasonal components, the sampling error, and the population white noise are defined in Subsection 3.1. To improve the precision of the nowcasts for the register series with the additional timely information from the LFS series, the correlation between the disturbance terms of the trend, seasonal component and population white noise, can be modelled in Equation (7). In the final selected model, only a non-zero correlation for the slope disturbance terms of the trend components is defined. The seasonal components turned out to be time invariant, while no correlation is detected between population white noise terms. This results in the following variance structure for the slope disturbances of the smooth trend models for the register and LFS series:

$$
\begin{gathered}
\operatorname{Cov}\left(\eta_{t, d}^{x}, \eta_{t^{\prime}, d}^{x}\right)=\left\{\begin{array}{ll}
\left(\sigma_{\eta, d}^{x}\right)^{2} & \text { if } t=t^{\prime} \\
0 & \text { if } t \neq t^{\prime}
\end{array} \text { for } x \in\{R, L F S\},\right. \\
\operatorname{Cov}\left(\eta_{t, d}^{R}, \eta_{t^{\prime}, d}^{L F S}\right)= \begin{cases}\sigma_{\eta, d}^{R} \sigma_{\eta, d}^{L F S} \rho_{\eta, d} & \text { if } t=t^{\prime} \\
0 & \text { if } t \neq t^{\prime}\end{cases}
\end{gathered}
$$

If the model detects (strong) correlation between the trends of both series, then this implies that the trends of both series develop more or less in the same direction. Note that Equation (8) defines a $2 \times 2$ covariance matrix for the slope disturbance terms of the trends. In the case of strong correlation, $\rho_{\eta, d} \rightarrow 1$ (or $\rho_{\eta, d} \rightarrow-1$ ), the rank of this covariance matrix will reduce to one. In that case, the trend components of both series are said to be cointegrated, since they are driven by one underlying common trend. Expressing the model in terms of a common factor model results in a more parsimonious model and improves the efficiency of the estimation procedure. See Harvey (1989, subsec. 8.5), or Koopman et al. (2007, subsec. 9.1), for more details concerning cointegration and common factor state space models.

In the case of strong correlation or even cointegration, the LFS series contains valuable information for the prediction of register labour force participation rates at times when those register data are not yet available while the LFS estimates have already been calculated. In the univariate case, the predictions are based on past observations of the register series alone. In the bivariate case, the correlation between both series may improve the accuracy of these predictions. To see whether more accurate small area estimates of the auxiliary series lead to greater improvements in the accuracy of the nowcasted main series results, the model calculations are repeated for a number of differently sized municipalities.

\subsection{Multiplicative Models}

Equations (3) and (7) are additive models. Since the target variables are percentages, multiplicative models can be considered as an alternative. This is achieved by taking the 
$\log$ of the observed series. Thus $y_{t, d}^{R}$ and $y_{t, d}^{L F S}$ are replaced by $\log \left(y_{t, d}^{R}\right)$ and $\log \left(y_{t, d}^{L F S}\right)$ in Equations (3) and (7) respectively. For the variance structure of the measurement equation of the LFS, an approximation of the MSE of the $\log \left(y_{t, d}^{L F S}\right)$ in Equation (6) is required. Based on a first-order Taylor approximation of $\log \left(y_{t, d}^{L F S}\right)$ it follows that $\operatorname{MSE}\left(\log \left(y_{t, d}^{L F S}\right)\right) \approx \operatorname{MSE}\left(y_{t, d}^{L F S}\right) /\left(y_{t, d}^{L F S}\right)^{2}$. In a similar way it follows for the auto-covariance that $\operatorname{cov}\left(\log \left(y_{t, d}^{L F S}\right), \log \left(y_{t-1, d}^{L F S}\right)\right) \approx \operatorname{cov}\left(y_{t, d}^{L F S}, y_{t-1, d}^{L F S}\right) /\left(y_{t, d}^{L F S} \times y_{t-1, d}^{L F S}\right)$. As a result, the autocorrelations are unaffected and the AR(1) structure derived for the additive model can be used for the multiplicative model.

\subsection{Estimation of Structural Time Series Models}

A widely applied approach to fit structural time series models is to put them in the socalled state space form. Under the assumption of normally distributed disturbance terms, optimal estimates of the state variables and linear combinations of these variables are obtained with the Kalman filter. State variables are the variables that define the unobserved components, that is, the $L_{t, d}^{x}$ and $R_{t, d}^{x}$ of the trend, the $\gamma_{j t, d}$ and $\tilde{\gamma}_{j t, d}$ of the seasonal component and the sampling errors $\tilde{e}_{t, d}^{L F S}$. The Kalman filter is a recursive procedure that runs from period $t=1$ to $T$ and gives, for each time period, an optimal estimate for the state variables based on the information available up to and including period $t$. These estimates are referred to as the filtered estimates. The filtered estimates of past state vectors can be updated, if new data after period $t$ become available. This procedure is referred to as smoothing and results in smoothed estimates that are based on the complete time series. In this article, the fixed-interval smoother is used. Variances for the state variables and signals are obtained with the standard Kalman filter recursions.

The Kalman filter assumes that the variances and covariances of the disturbance terms in the models for the trend, seasonal components, sampling error and population white noise, are known in advance. These components are often referred to as the hyperparameters of the state space model. In practice, these parameters are not known and therefore have to be estimated. In this article, maximum likelihood estimates for the hyperparameters are obtained by numerically optimising the likelihood function with the Broyden-Fletcher-Goldfarb-Shanno (BFGS) algorithm and repeatedly running the Kalman filter.

The maximum likelihood estimates for the hyperparameters are inserted in the Kalman filter. The uncertainty due to replacing the unknown hyperparameter values for their maximum likelihood estimates is further ignored in the standard errors of the Kalman filter estimates for the state variables and signals. Pfeffermann and Tiller (2005) developed a bootstrap to account for the additional uncertainty of the maximum likelihood hyperparameter estimates in the standard errors of the Kalman filter estimates. An alternative approach is to express the bivariate model as an hierarchical Bayesian time series multilevel model and fit the model using MCMC simulations. This approach also accounts for the additional uncertainty of the hyperparameter estimates (see, for example, Boonstra and Van den Brakel 2019). Bollineni-Balabay et al. (2017) analysed the additional uncertainty in the standard errors of the Kalman filter estimates in the Dutch LFS because maximum likelihood estimates for the hyperparameters are plugged into the Kalman filter recursions. They conclude that this additional increase is ignorable in their 
application. In this article, we follow the standard state-space approach and ignore this uncertainty in this application.

The Kalman filter recursions provide predictions for missing observations in the time series. These predictions can be interpreted as imputations obtained by the EM algorithm (Durbin and Koopman 2012, subsubsec. 7.3.4). This property is used here for nowcasting the register series. The last four quarters of the register series are missing. The Kalman filter provides predictions or nowcasts for these missing values, including their variances, which can be interpreted as imputations obtained by the EM algorithm.

All state variables, except for the sampling error $\tilde{e}_{t, d}^{L F S}$ of the LFS series, are nonstationary. For the non-stationary state variables a diffuse initialization of the Kalman filter is used. This means that their starting values at $\mathrm{t}=0$ are chosen equal to zero with a large variance (10E7). The sampling errors are stationary and for their state variables an exact initialization can be applied. This means that their starting values are chosen equal to zero with a variance derived from the assumed AR(1) process. More technical details about state space models and their analysis can be found in Harvey (1989) or Durbin and Koopman (2012).

Performance of the models for nowcasting register labour force participation out of sample forecasts is evaluated for the periods 2012 through 2014. Let T denote the quarter of the last year that is observed with the register. For $\mathrm{T}$ equal to the fourth quarter of respectively 2011, 2012 and 2013, out of sample forecasts for the state variables and signals for the register employed labour force are made for the next four quarters, that is, $\mathrm{T}+1, \mathrm{~T}+2, \mathrm{~T}+3$ and $\mathrm{T}+4$. Results for nowcasts are estimated in real time, that is, the hyperparameters are re-estimated as a new observation becomes available.

Model selection is based on likelihood diagnostics like AIC (Durbin and Koopman 2012, subsec. 7.4) and likelihood-ratio tests (Harvey 1989, subsec. 5.1) and an evaluation of the model assumptions. The likelihood ratio test is used to test restrictions on hyperparameters, for example, to test whether the correlation between the slope disturbance terms in the bivariate model are equal to zero and to whether the variance of the level disturbance terms of the local linear trend model equals zero and thus can be restricted to the smooth trend model. The AIC criteria are used to compare non-nested models, for example, to compare the smooth trend model with the local level model. The state space models considered here assume that all disturbance terms are normally and independently distributed. As a result, the one-step-ahead predictions or innovations should also be normally and independently distributed. The model assumptions can be checked by performing a number of model diagnostics on the standardised innovations: calculation of mean, variance, skewness, kurtosis, Bowman-Shenton-normality test, Ljung-Box test on serial correlation for the first twelve lags, Durbin-Watson test on serial correlation (Durbin and Koopman 2012, subsec. 2.12) and a visual check on outliers, by plotting the standardised innovations. Also, an F-test has been used on the first and last twelve innovations in each of the series to test for heteroscedasticity (Durbin and Koopman 2012, 39).

The time series models are implemented in the OxMetrics software package, in combination with subroutines of SsfPack 3.0. For more information, see Doornik (2009) and Koopman et al. (2008). 


\section{Results}

\subsection{Model Parameters for Univariate and Bivariate Models}

The univariate and bivariate analyses are based on the models specified in Subsections 3.1 and 3.2. They are applied to two series: the series of LFS municipal small area predictions using the Battese-Harter-Fuller unit level model, as described in Subsection 2.1, and a register series based on tax service data sources that include persons with paid employment and self-employment. The LFS and register series run from the first quarter in 2003 up to and including the final quarter of 2014. Due to the diffuse initialisation of five state variables in the Kalman filter, the first six quarters of the time series are removed from the presentation of the results to allow the Kalman filter to converge to a stable distribution for the state variables. Time series results, therefore, start at the third quarter of 2004 and run up to and including the last quarter of 2014. This period contains 42 quarters.

The performance of the proposed method to estimate the register series is evaluated over the last three years of the observed series in real time. Producing nowcasts in real time is achieved as follows. To obtain nowcasts for the register series for 2012, models are fitted to register series observed until the last quarter of 2011. With the univariate model, applied to the register series only, predictions are made for the four quarters of 2012 based on the register series observed until the last quarter of 2011. With the bivariate model, nowcasts for the four quarters of 2012 are based on the register series observed until the last quarter of 2011 and the LFS observed until the particular quarter of 2012 for which a nowcast of the register is calculated. This process is repeated for 2013 and 2014 using the register series observed until the last quarter of 2012 and 2013 respectively.

Variances and covariances of the innovations, and results for the model diagnostics, in order to establish that the innovations are normally and independently distributed, are included in Appendix (Subsection 6.2) for both the univariate and bivariate models. We also mention that plots of the standardised innovations do not indicate that there are outliers. These diagnostics show that no severe deviations of the underlying model assumptions exist. The maximum likelihood estimates for the additive univariate and bivariate model hyperparameters are presented in Tables 1 and 2 . The hyperparameters are variances and are estimated on the log-scale to avoid negative maximum likelihood estimates. As a result, the confidence intervals are asymmetrical. Both the register and the LFS series are at the municipal level.

The small values for the variance of the seasonal disturbance terms for the register series in both the univariate and bivariate model illustrate that the seasonal component hardly changes over time. This also holds for the variance of the seasonal disturbance terms for

Table 1. Maximum likelihood estimates hyperparameters register univariate model (full series) with $95 \%$ confidence interval between brackets.

\begin{tabular}{llll}
\hline Hyperparameter & Amsterdam & Heerlen & Haren \\
\hline Trend $\left(\sigma_{\eta, d}^{R}\right)$ & $0.135(0.091-0.201)$ & $0.157(0.108-0.231)$ & $0.150(0.085-0.267)$ \\
Seasonal $\left(\sigma_{\omega, d}^{R}\right)$ & $0.027(0.014-0.055)$ & $0.014(0.005-0.049)$ & $0.027(0.011-0.067)$ \\
Measurement & $0.128(0.084-0.197)$ & $0.140(0.097-0.202)$ & $0.209(0.140-0.280$ \\
$\quad$ equation $\left(\sigma_{\xi, d}^{R}\right)$ & & & \\
\hline
\end{tabular}


Table 2. Maximum likelihood estimates hyperparameters bivariate model (full register series) with $95 \%$ confidence interval between brackets.

\begin{tabular}{llll}
\hline Hyperparameter & Amsterdam & Heerlen & Haren \\
\hline Trend & 0.134 & 0.155 & 0.139 \\
$\quad$ register $\left(\sigma_{\eta, d}^{R}\right)$ & $(0.091-0.197)$ & $(0.106-0.227)$ & $(0.077-0.250)$ \\
Seasonal & 0.027 & 0.014 & 0.026 \\
$\quad$ register $\left(\sigma_{\omega, d}^{R}\right)$ & $(0.013-0.054)$ & $(0.004-0.050)$ & $(0.011-0.067)$ \\
Measurement equation & 0.131 & 0.142 & 0.220 \\
$\quad$ register $\left(\sigma_{\xi, d}^{R}\right)$ & $(0.086-0.198)$ & $(0.099-0.206)$ & $(0.147-0.329)$ \\
Trend & 0.112 & 0.128 & 0.103 \\
$\quad$ LFS $\left(\sigma_{\eta, d}^{L F S}\right)$ & $(0.084-0.157)$ & $(0.089-0.185)$ & $(0.047-0.244)$ \\
Seasonal & $<0.001$ & 0.014 & 0.026 \\
$\quad$ LFS $\left(\sigma_{\omega, d}^{L F S}\right)$ & $(0.000-9.870)$ & $(0.001-2.309)$ & $(0.002-0.384)$ \\
Measurement & 0.231 & $<0.001$ & $<0.001$ \\
$\quad$ eq. $L F S\left(\sigma_{\xi, d}^{L F S}\right)$ & $(0.057-0.940)$ & $(0.000-1.984)$ & $(0.000-9.430)$ \\
Corr. slope & 0.899 & 0.999 & 0.980 \\
$\quad$ residuals $\left(\rho_{\eta, d}\right)$ & & & \\
Survey error & 0.758 & 0.766 & 0.578 \\
$\quad$ AR $(1)-$ noise $\left(\sigma_{s, d}\right)$ & $(0.408-1.408)$ & $(0.612-0.959)$ & $(0.462-0.702)$ \\
\hline
\end{tabular}

the LFS series. The variance of the slope disturbance terms for the register series and the LFS series are large enough to give the trend the flexibility to accommodate for cyclic movements, as can be seen for the LFS series in Figure 3. For the register series, the variance of the measurement errors are about the same size as the variances of the slope disturbance terms. For the LFS series, this is only true for Amsterdam. For the other two cities the variance of the measurement errors are almost zero. A possible reason for this is that the sampling error in the two smaller cities (Heerlen and Haren) is much larger than the capital city Amsterdam. It appears that the sampling error dominates the uncertainty in the series of Heerlen and Haren and that the population white noise is absorbed in the sampling error component. The variance estimates for the three components (trend, seasonal and measurement error) of the register series under the univariate model in Table 1 are almost similar to the values under the bivariate model in Table 2.

The AR(1) autoregressive parameter for the sampling errors in the LFS series is estimated from the micro data as described in Subsection 3.1 (its value is 0.59). Recall from Subsection 3.1 that we expect values for the survey error $\operatorname{AR}(1)$ noise that are close to $\sigma_{s, d}^{2} \approx\left(1-\rho_{d}^{2}\right)$, which implies that $\sigma_{s, d} \approx 0.8$. This holds reasonably for Amsterdam and Heerlen. The value for Haren is clearly smaller, which implies that the model reduces the MSE estimates for the BHF domain predictions of the LFS input series. The interpretation of the time series model is that the volatility of the LFS input series is smaller than the MSE estimates of the LFS domain predictions simply. One possible reason is that the BHF unit level model over shrinks the domain estimates, in particular for a small municipality like Haren.

In this application, the results obtained with the additive model are very similar to the results obtained under the multiplicative model. In Figure 3, the results under the bivariate additive and bivariate multiplicative models are compared for Heerlen. The confidence interval for the multiplicative model is asymmetric due to the anti-log transformation, but even this is hardly visible. Also, the model diagnostics are very similar under both models. 


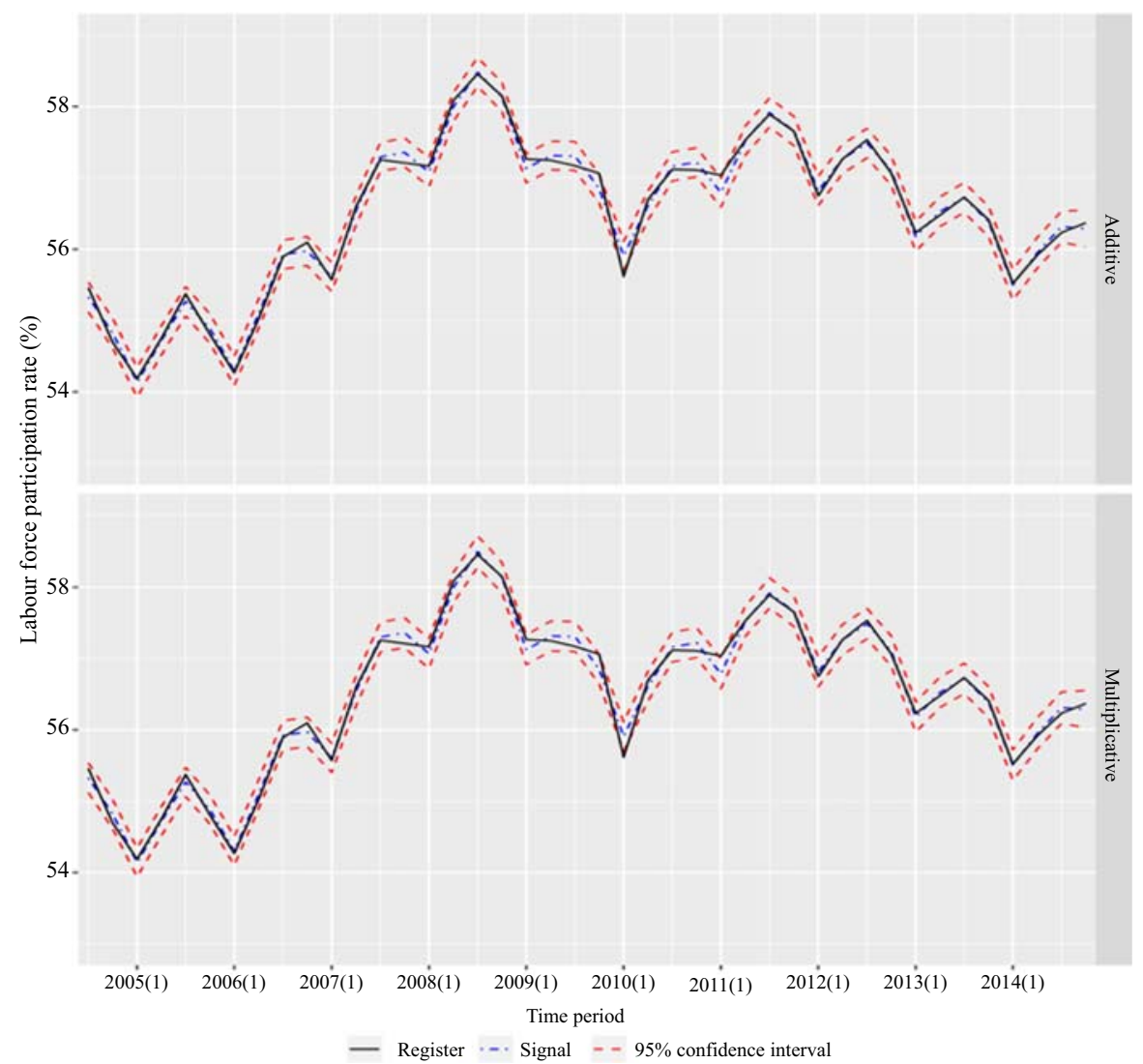

Fig. 3. Comparison Additive and Multiplicative Bivariate Model for Heerlen.

In Appendix (Subsection 6.2) the model diagnostics for the univariate and bivariate multiplicative model are included. In what follows, additive models are used.

The bivariate model detects a strong correlation between the LFS and register slope components in the bivariate analyses. A likelihood ratio test has been applied to test the significance of the correlation between the slope disturbances. If the correlation parameter is set to zero, the likelihood is reduced significantly. For the municipalities Amsterdam, Heerlen and Haren, the p-values of the corresponding likelihood ratio tests are 0.0021, 0.0025 , and 0.0500 respectively.

\subsection{Results for Univariate and Bivariate Models at Municipal Level}

In Figures 4 and 5, two examples are presented of the nowcasting analyses of the univariate and bivariate models for the register series. The figures compare the nowcasts estimated in real time under the univariate and bivariate model with the final observations from the register. For both time series models, three series are compared; one based on the register information available up until the end of 2012, one based on the register information available up until the end of 2013, and one based on the register information available up until the end of 2014. The three time series for the univariate model are plotted in red, while the three time series of the bivariate model are plotted in blue. The end of the time series 


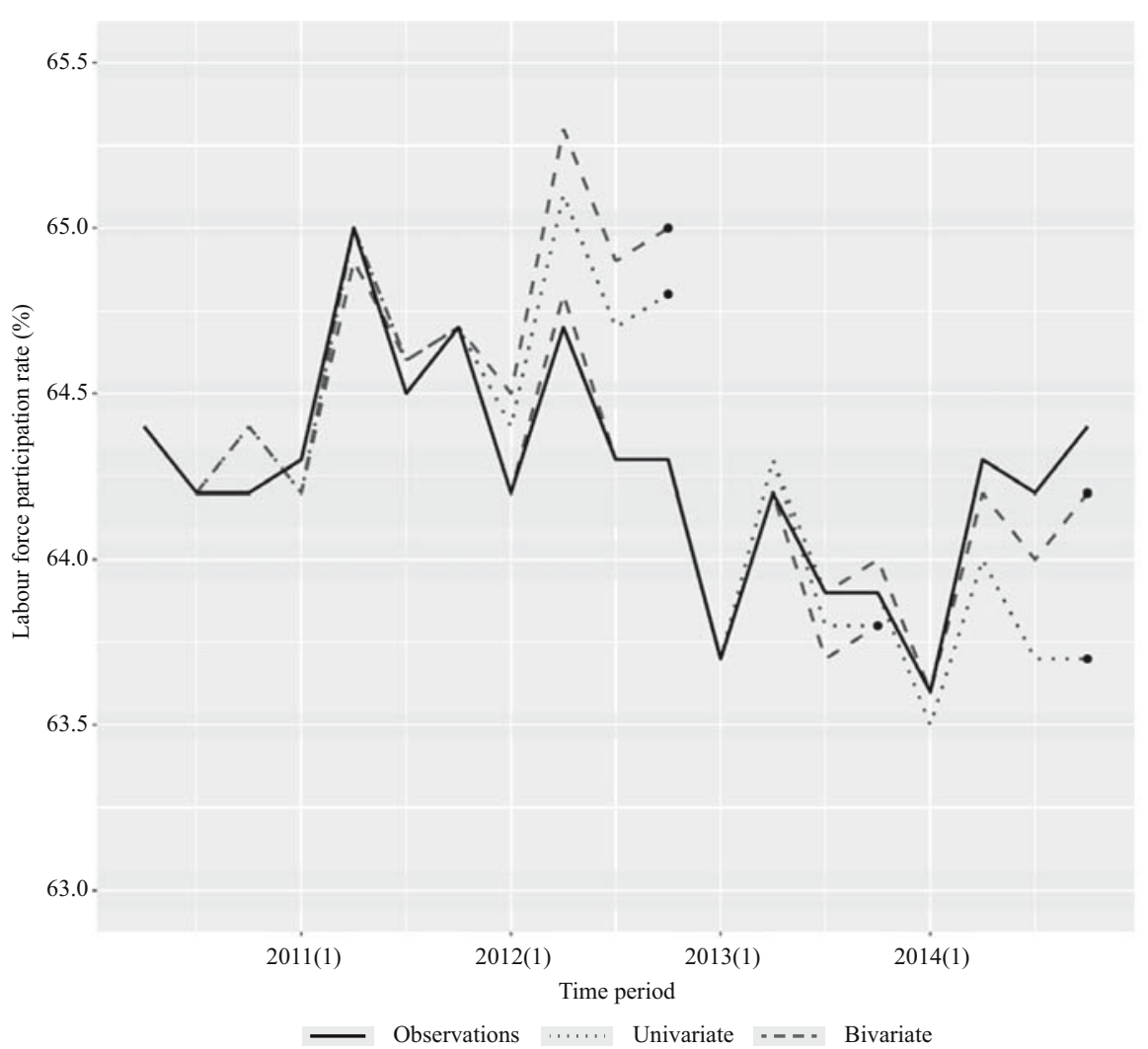

Fig. 4. Amsterdam: Smoothed signal of the labour force participation rate for the register series in the univariate and bivariate models with nowcasts for the last four measurements, compared to the final observations from the register.

estimates under both models are indicated with a red or blue dot. This in real time analysis results give an indication of the size of the revisions if new data become available afterwards.

Both the univariate and bivariate model results show nearly identical smoothed time series up to the last four quarters of 2014. However, for the last four quarters, where onestep, two-step, three-step and four-step ahead predictions were made, the results from the univariate and bivariate analysis differ. For the case of Amsterdam, the bivariate analysis nowcasts for 2014 are closer to the smoothed results of the full series than the nowcasts of the univariate analysis. In the case of Haren (Figure 5), a small municipality in the north of the Netherlands, the results lead to the opposite conclusion.

In Figure 6, standard errors based on the univariate and bivariate models are compared for the smoothed and nowcasted signal of the labour force participation rates in Amsterdam, Heerlen and Haren. For reference, the standard errors of the small area estimates of the LFS labour force participation rates are also presented.

The gain in accuracy obtained with the bivariate model with respect to the univariate model is approximately 20\% in Amsterdam, Heerlen and Haren for 2014. For Haren, in 2012 and 2013 there is hardly any difference. The smaller gain in accuracy for Haren may be related to the smaller correlation between the register and LFS slope disturbance terms. However, the variance of the LFS small area estimates may also be a contributing factor. For Amsterdam, 


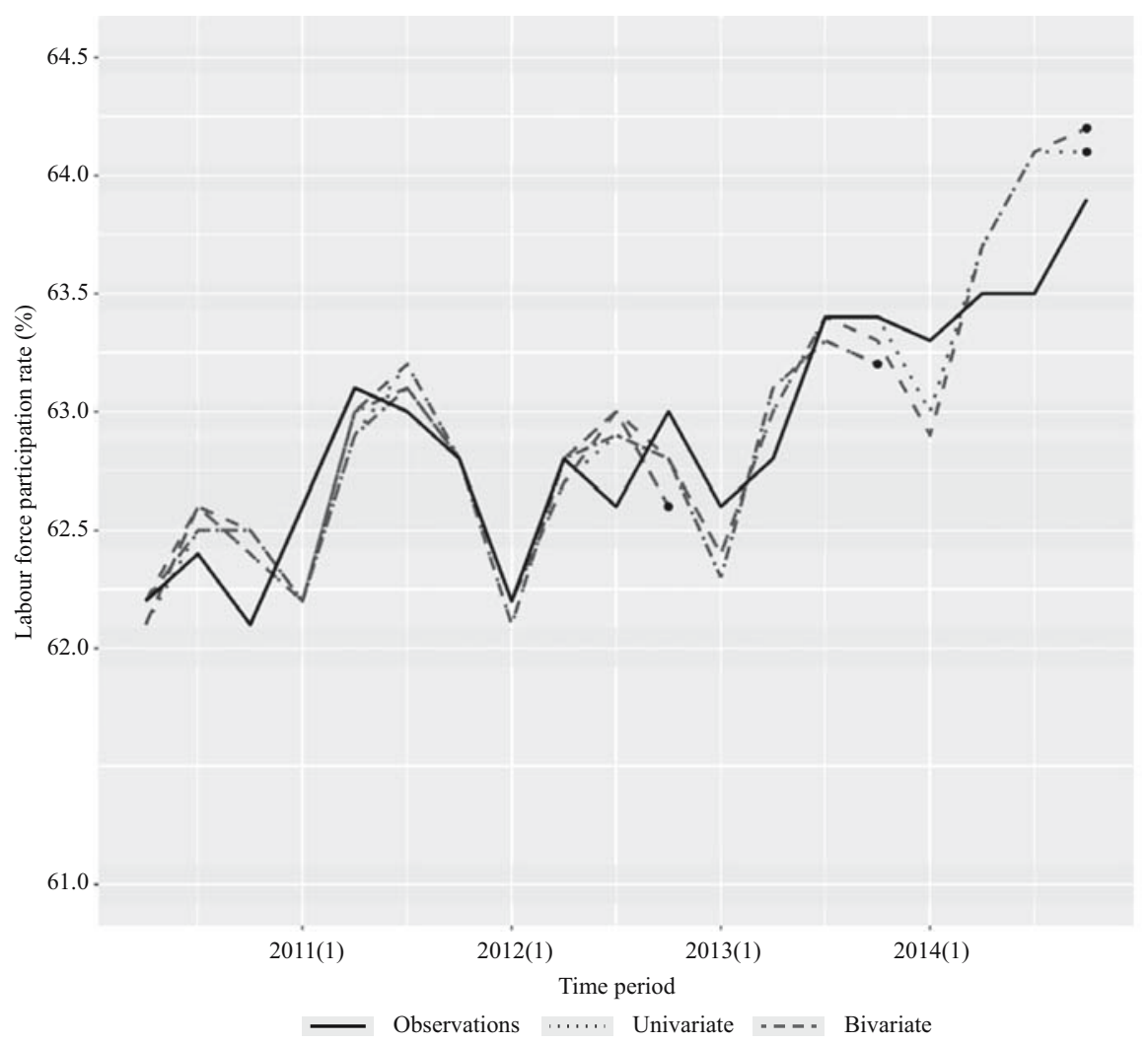

Fig. 5. Haren: Smoothed signal of the labour force participation rate for the register series in the univariate and bivariate models with nowcasts for the last four measurements, compared to the final observations from the register.

the standard errors of the time series nowcasts are comparable with the standard errors of the LFS small area predictions. For smaller municipalities, the nowcasted results of the register series are more accurate than the LFS estimates of the labour force participation rate.

As a second analysis, the time series of the GREG estimates of the labour force participation rate at the national level is used as an auxiliary series to nowcast the Haren register series. Although the correlation between these two series is smaller $(0.783)$, the reduction in standard error in going from the univariate to the bivariate analysis is clearly larger: 39\%. The relatively small standard errors of the national LFS estimates of the labour force participation rate seem to be an important contributing factor; they are much smaller than those of the Haren LFS auxiliary series ( $0.15 \%$ point instead of $1.4 \%$ point).

This suggests that at least for small municipalities, where the variance of the LFS series is large and the correlation between the register series and the national LFS estimates is not too small, it may be better to use the national LFS series instead of the municipal series. Using auxiliary series at high aggregation levels, for example, national or province level, however, might introduce larger amounts of bias in the model predictions.

In a next step, the univariate and bivariate models are applied to all municipalities in the Netherlands. The estimated standard errors of the predicted signals of both the univariate and bivariate time series models are further evaluated with the coverage rates of the 


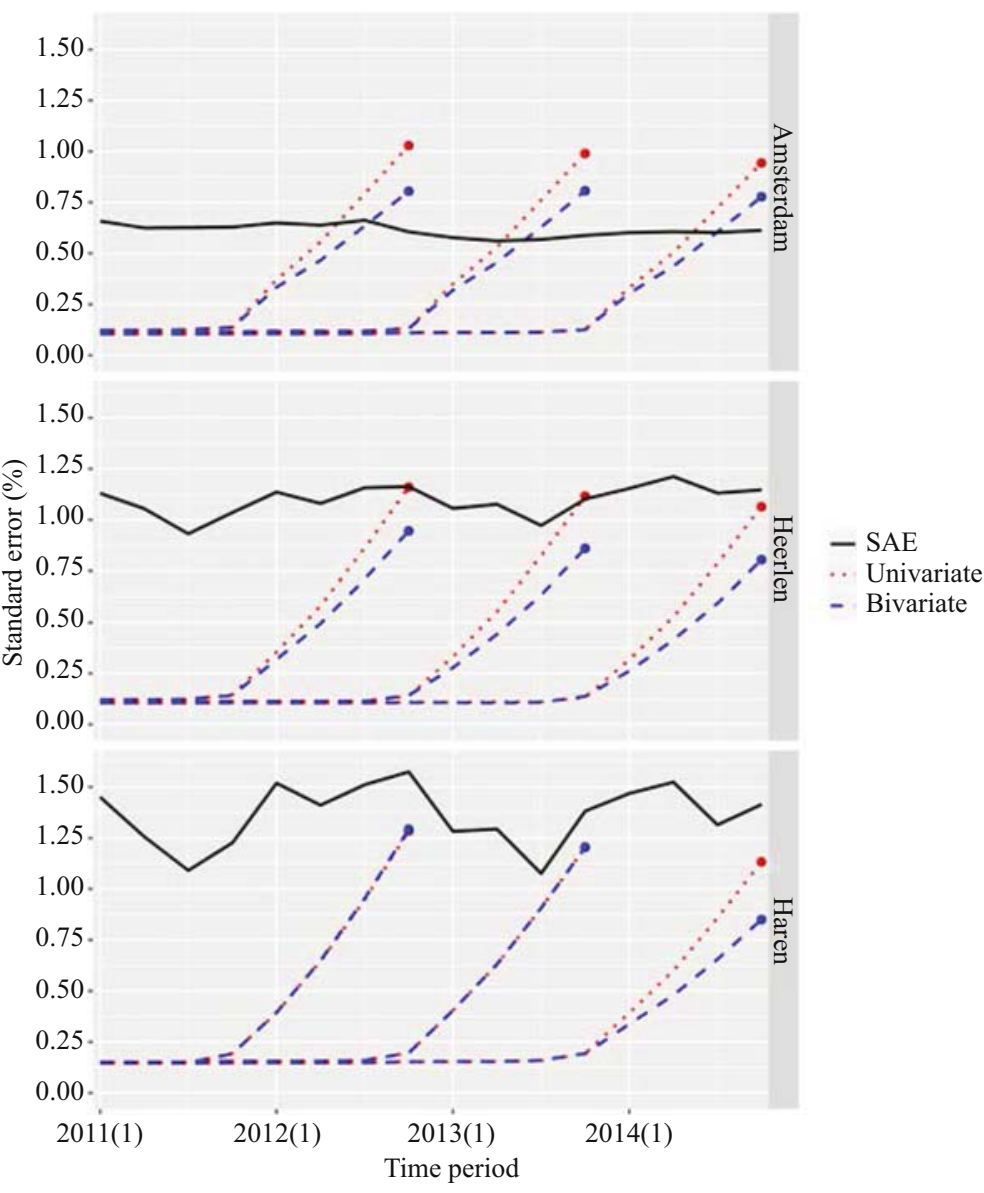

Fig. 6. Standard errors of predicted signal of the municipal labour force participation rate for the register series in the univariate and bivariate models compared with standard errors of small area estimates (nowcasting in each of the last three years of the time series).

corresponding $95 \%$ confidence intervals. For both time series models, the proportion of municipalities for which the measured value of the labour force participation rate is to be found within the estimated 95\%-confidence interval is calculated. The results for the 2014 nowcasts are presented in Table 3.

Table 3. Coverages of the one-step to four-step ahead predictions of the register labour force participation rate (municipalities) for the univariate and bivariate time series models, 2014 (nowcasts for all municipalities in the Netherlands).

\begin{tabular}{lccc}
\hline Type of estimate & $\begin{array}{c}\text { Univariate } \\
\text { \% }\end{array}$ & Bivariate $^{\mathbf{1}}$ & Bivariate $^{\mathbf{2}}$ \\
\hline 1-step ahead & 95.8 & 92.6 & 90.0 \\
2-step ahead & 99.0 & 95.1 & 94.9 \\
3-step ahead & 99.3 & 95.3 & 96.3 \\
4-step ahead & 99.3 & 94.9 & 97.5 \\
\hline
\end{tabular}

${ }^{1}$ using municipal small area predictions as auxiliary series.

${ }^{2}$ using national GREG estimates as auxiliary series. 
Table 4. Root relative mean square error of the one-step to four-step ahead predictions of the register labour force participation rate (municipalities) for the univariate and bivariate time series models, 2014 nowcasts.

\begin{tabular}{lccc}
\hline RRMSE & $\begin{array}{c}\text { Univariate } \\
\text { \% }\end{array}$ & Bivariate $^{\mathbf{1}}$ & Bivariate $^{\mathbf{2}}$ \\
\hline 1-step ahead & 0.54 & 0.55 & 0.56 \\
2-step ahead & 0.72 & 0.68 & 0.66 \\
3-step ahead & 0.95 & 0.91 & 0.81 \\
4-step ahead & 1.31 & 1.19 & 1.04 \\
\hline
\end{tabular}

${ }^{1}$ using municipal small area predictions as auxiliary series.

${ }^{2}$ using national GREG estimates as auxiliary series.

It turns out that the confidence regions of the univariate nowcasts are approximately equal to $95 \%$ for the one-step-ahead prediction but are too wide for the two-, three- and four-step ahead predictions. For the bivariate time series model (1) the confidence intervals are too small for the one-step-ahead predictions and approximately correct for the two-, three- and four-step ahead predictions. For the bivariate model (2) only the two-step ahead predictions are approximately correct.

To compare the accuracy of estimates obtained with the univariate and bivariate model, the root relative mean square errors (RRMSE's) are calculated:

$$
R R M S E_{t, j}=100 \% \times \frac{1}{n_{\text {mun }}} \sqrt{\sum_{n_{\text {mun }}}^{d=1}\left(\frac{y_{d, t+j \mid t}^{R}-y_{d, t+j \mid t+4}^{R}}{y_{d, t+j \mid t+4}^{R}}\right)^{2}}
$$

with $y_{d, t+j \mid t}^{R}$ for $j=1, \ldots, 4$, the prediction or nowcast for the register labour force participation rate in municipality $d$ for period $t+j$ based on the information observed until period $t, y_{d, t+j \mid t+4}^{R}$ the register value for the register labour force participation rate in municipality $d$ as it eventually becomes available, and $n_{\text {mun }}$ the number of municipalities. In Table 4, the RRMSEs of the 2014 nowcasts for the univariate and bivariate models are presented. Using this measure, the register estimates for the labour force participation rate in the bivariate model are more accurate than the univariate estimates (except for the first step ahead prediction), although the gain in accuracy seems less than as suggested by the estimated standard errors. Furthermore, these results show that using the national LFS labour force participation rates as an auxiliary series gives better estimates for the nowcasts.

\subsection{Results for Univariate and Bivariate Models at Neighbourhood Level}

In this subsection, the univariate and bivariate modelling approach for nowcasting the register labour force participation rates is extended to the level of neighbourhoods. As it is not practical to perform this kind of analysis on all neighbourhoods, municipality Heerlen is selected as an example and several of its neighbourhoods are considered in the analysis. In Table 5, the selected neighbourhoods from Heerlen together with a number of their characteristics are presented.

Figure 7 compares the measured labour force participation rates for several Heerlen neighbourhoods (indicated by 'Observations') with the predicted signals of the bivariate time series analysis (indicated by 'Bivariate'. Similar to Figures 4 and 5, this figure 


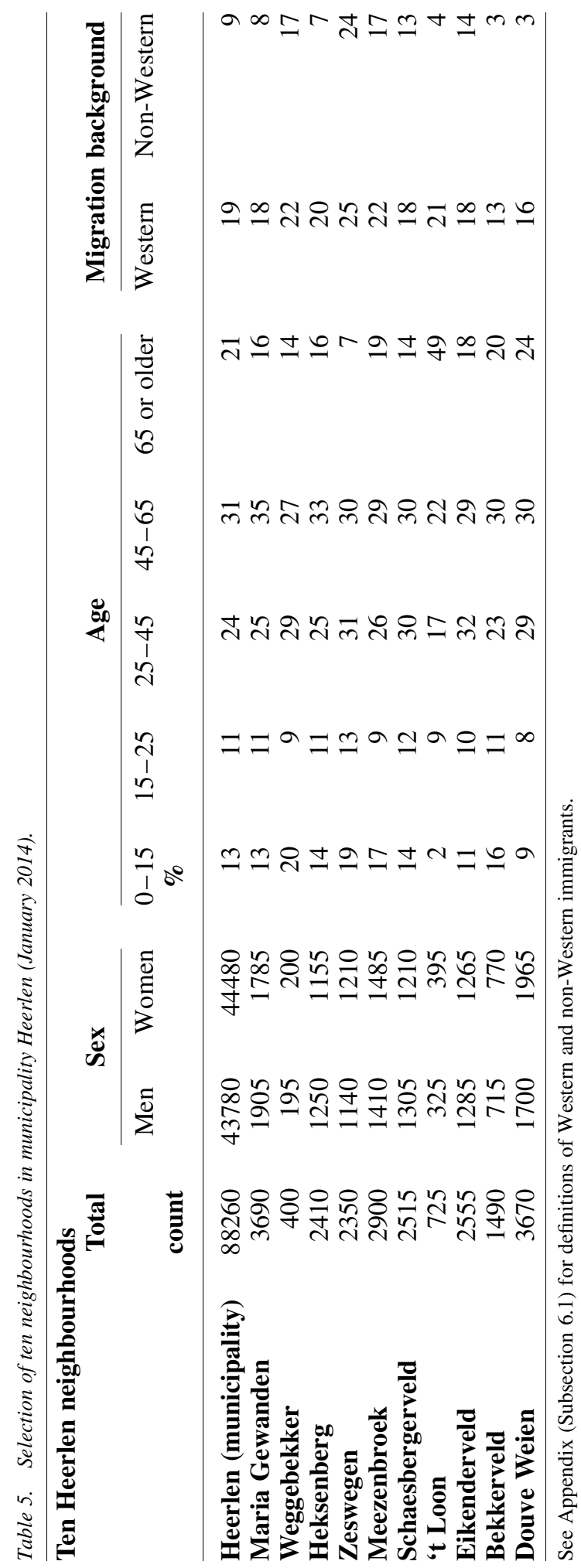




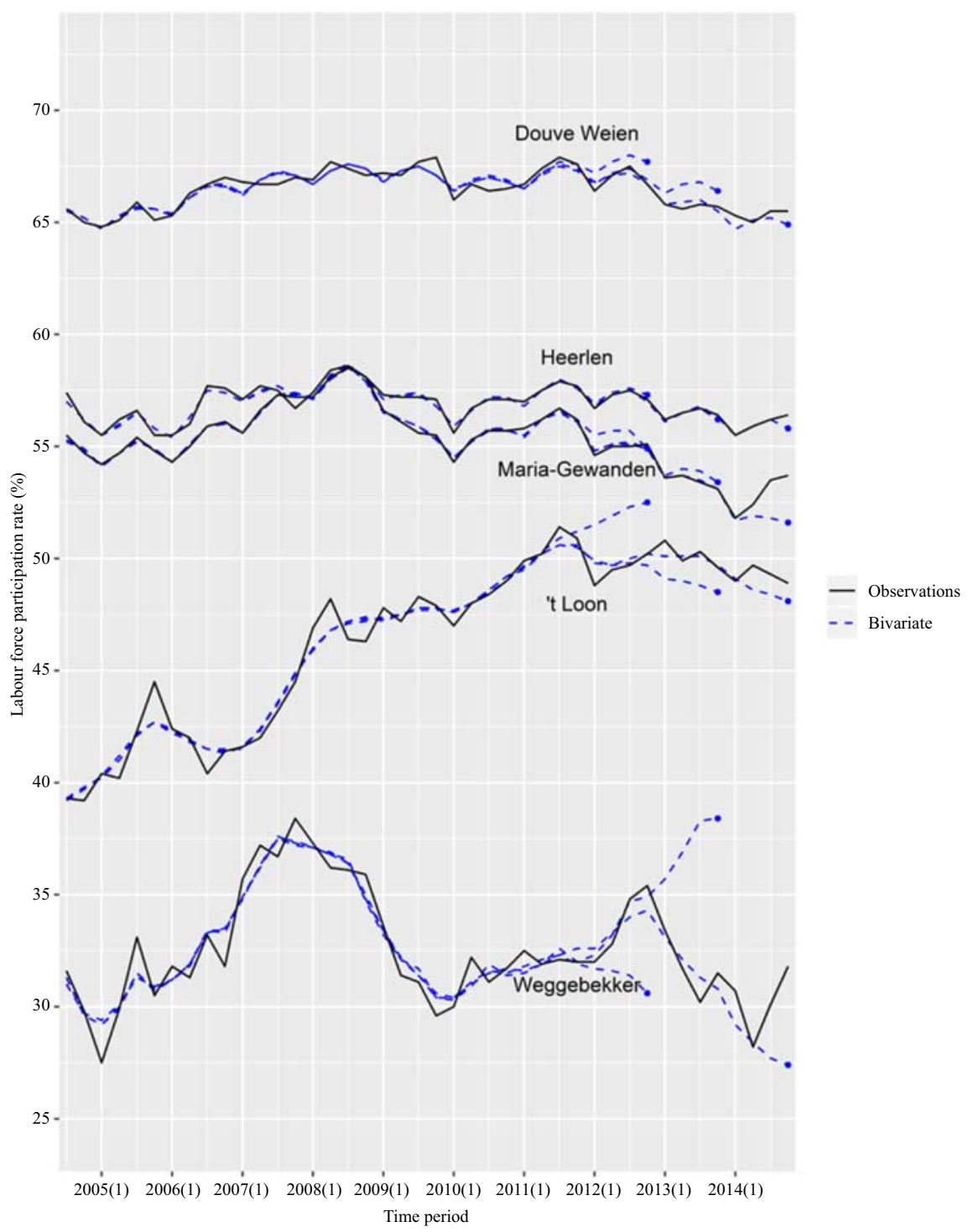

Fig. 7. Time series labour force participation rates for Heerlen and several of its neighbourhoods, comparing register measurements with predicted signals from the bivariate model (series run from third quarter of 2004 to the last quarter of 2014, with observations for each of the last three years).

compares the nowcasts estimated in real time using the bivariate model with the final observations from the register. To this end, three series of time series estimates are compared; one based on the information available up until the end of 2012, one based on the information available up until the end of 2013, and one based on the information available up until the end of 2014. The end of the time series estimates under the bivariate model are indicated with a blue dot. For some neighbourhoods the nowcasts are close to the final values of the register, for example, in the case of Douve Weien and Maria Gewanden. These neighbourhoods typically follow the municipal pattern. For other neighbourhoods, for example, Weggebekker, the model fails to predict unexpected turning 
points. This might be explained by the fact that Weggebekker is a small and rather atypical Heerlen neighbourhood. It contained 365 inhabitants in 2018, which partly explains the volatility of the labor force participation rates over the years; also other characteristics deviate from the municipal average (e.g., in 2018: home ownership is only 5\% compared to $46 \%$ in all of Heerlen; and the percentage of inhabitants with social benefits due to disability or unemployment is $30 \%$, compared to $16 \%$ in Heerlen municipality).

In order to evaluate the accuracy of the univariate and bivariate nowcasts for these neighbourhoods, a number of comparisons have been produced. First, like in Subsection

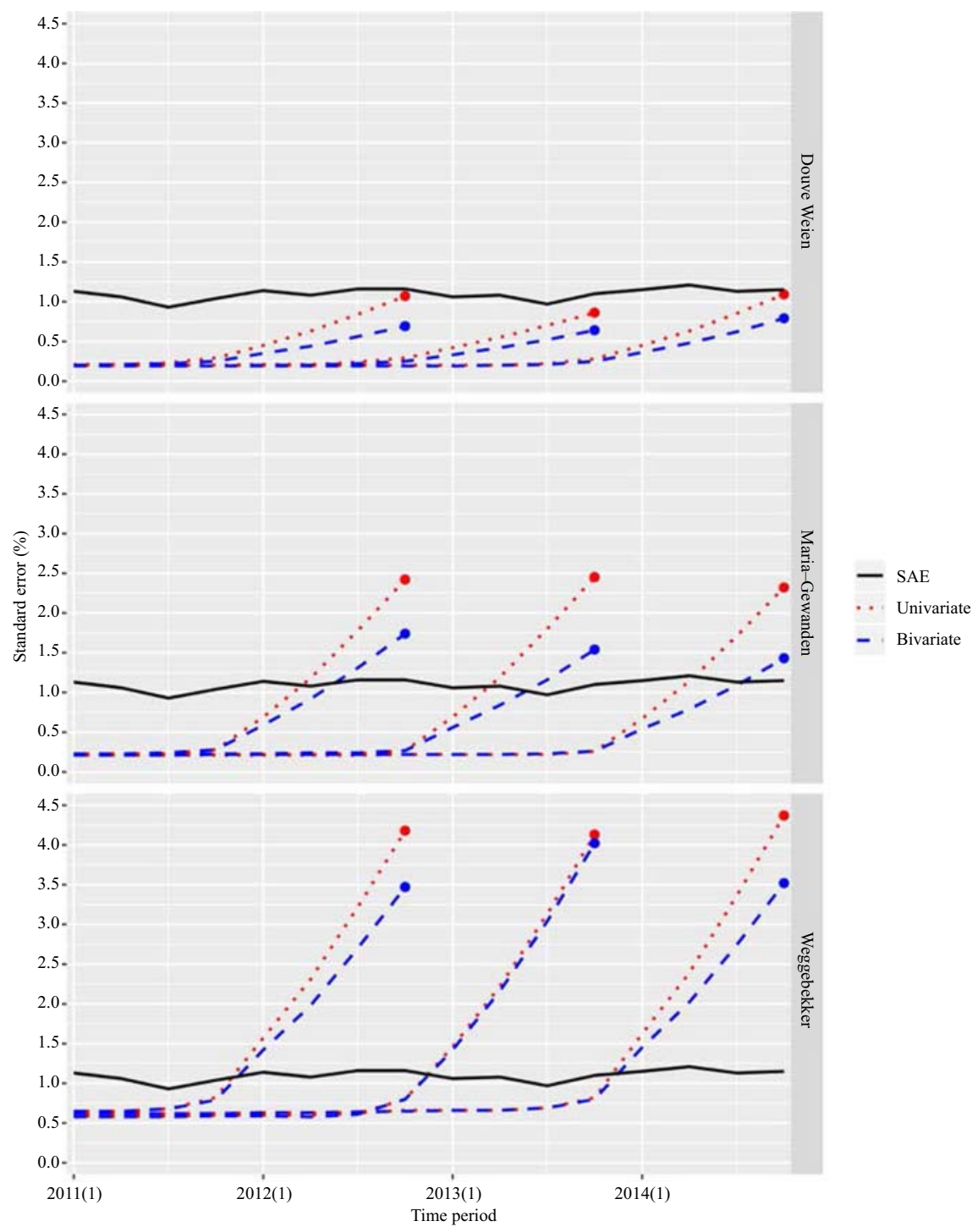

Fig. 8. Standard errors of the predicted signals of neighbourhood labour force participation rates for the register series at the neighbourhood level in the univariate and bivariate models compared with standard errors of small area estimates at the corresponding municipal level (nowcasting in each of the last three years of the time series). Results are shown for three neighbourhoods within municipality Heerlen. 
4.2, the standard errors of the nowcasts obtained with both the univariate and bivariate time series models are compared with one another, in this case for several Heerlen neighbourhoods (see Figure 8). For reference, the standard errors of the LFS small area estimates for municipality Heerlen, obtained with the unit-level model, are included in the presentation. As with the municipal series, the standard errors obtained with the bivariate models for the Heerlen neighbourhoods are smaller than the standard errors of the corresponding univariate models. The standard errors for the nowcasts of the two larger neighbourhoods (Douve Weien and Maria-Gewanden) are of the same order of magnitude as the standard errors of the LFS municipal small area estimates for Heerlen. For neighbourhood Weggebekker, a small neighbourhood, the standard errors of both the univariate and bivariate analyses are relatively large (factor three to four times the standard errors of the LFS municipal small area estimates).

In Table 6, the coverage rates of the $95 \%$ confidence intervals for the neighbourhood nowcasts in 2014 are compared for several models: the univariate model, and the bivariate model where either the LFS series for Heerlen is used or the LFS series at the national level. Coverage rates for all three approaches are smaller than expected. This might be a result of the erratic behaviour of the register series at the very detailed level of neighbourhoods.

In Table 7, the RRMSE for the nowcasts of the Heerlen neighbourhoods in 2014 are compared for the univariate and the bivariate models. The smallest RRMSEs are obtained with the bivariate model where the register series is combined with the LFS series at the national level. The results for the bivariate model with municipal small area estimation predictions as auxiliary variables are comparable with the results obtained with the univariate model.

Table 6. Coverages of the one-step to four-step ahead predictions of the register labour force participation rate in Heerlen neighbourhoods for the univariate and bivariate time series models, 2014 nowcasts.

\begin{tabular}{lccc}
\hline Type of estimate & $\begin{array}{c}\text { Univariate } \\
\mathbf{\%}\end{array}$ & Bivariate $^{\mathbf{1}}$ & Bivariate $^{\mathbf{2}}$ \\
\hline 1-step ahead & 85.7 & 83.9 & 82.1 \\
2-step ahead & 80.4 & 80.4 & 75.0 \\
3-step ahead & 85.7 & 87.5 & 71.4 \\
4-step ahead & 92.9 & 85.7 & 82.1 \\
\hline
\end{tabular}

${ }^{1}$ using municipal small area predictions as auxiliary series.

${ }^{2}$ using national GREG estimates as auxiliary series.

Table 7. Root relative mean square error of the one-step to four-step ahead predictions of the register labour force participation rates in Heerlen neighbourhoods for the univariate and bivariate time series models, 2014 nowcasts.

\begin{tabular}{lccc}
\hline RRMSE & $\begin{array}{c}\text { Univariate } \\
\text { \% }\end{array}$ & Bivariate $^{\mathbf{1}}$ & Bivariate $^{\mathbf{2}}$ \\
\hline 1-step ahead & 4.78 & 5.38 & \\
2-step ahead & 5.67 & 5.67 & 4.67 \\
3-step ahead & 6.28 & 6.26 & 4.18 \\
4-step ahead & 5.76 & 6.24 & 4.55 \\
\hline
\end{tabular}

${ }^{1}$ using municipal small area predictions as auxiliary series.

${ }^{2}$ using national GREG estimates as auxiliary series. 


\subsection{Nowcasting During the COVID-19 Pandemic}

An important issue with nowcasting is how accurate turning points are detected. To evaluate how well the nowcasts with the bivariate model pick up turning points in the target series, the nowcast exercise is extended to the COVID-19 pandemic for the two municipalities Amsterdam and Heerlen. The municipality Haren merged with another large municipality (Groningen) in 2019 and is therefore left out of this analysis. The Netherlands went into a lockdown at 16 March 2020. Any effect of the lockdown on the employed labour force will therefore be visible from the second quarter of 2020 onwards. The auxiliary series for the LFS are available up to the second quarter of 2020. The register series are available up to the fourth quarter of 2018. This implies that nowcasts are produced for the last six quarters. Nowcasts are calculated using the bivariate state space model with the LFS municipal small area predictions and the LFS GREG estimates at the national level being used as auxiliary series.

In Figure 9, the nowcasts of the register series for Amsterdam and Heerlen obtained with the bivariate model and the LFS municipal small area predictions as an auxiliary series, are compared with the LFS municipal small area prediction and the register series observed up to the fourth quarter of 2018. In Figure 10, the nowcasts of the register series for Amsterdam and Heerlen obtained with the bivariate model and GREG estimates of the LFS at the national level as the auxiliary series are compared with the LFS municipal small

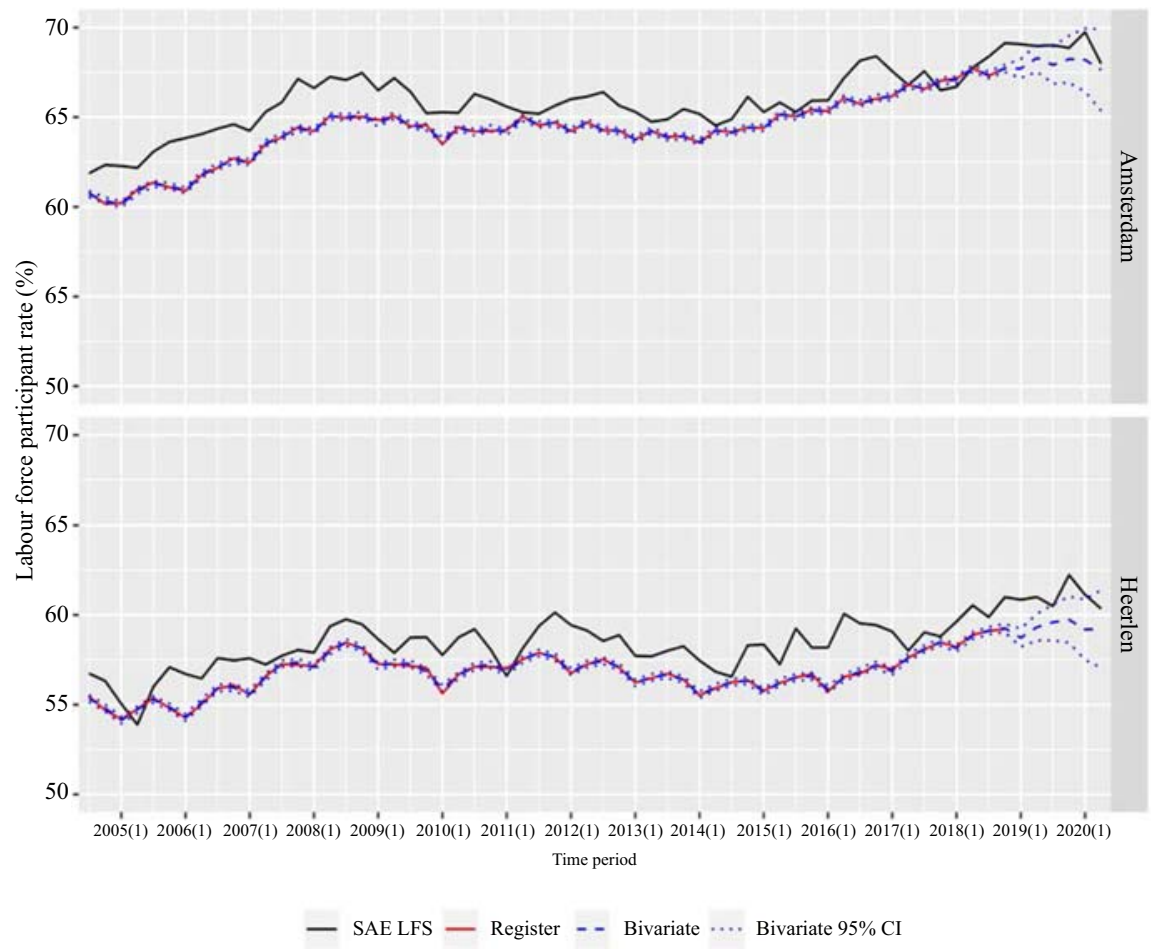

Fig. 9. Nowcasting the register series in the COVID-19 pandemic for the municipalities Amsterdam and Heerlen (municipal small area estimates as auxiliary series). 


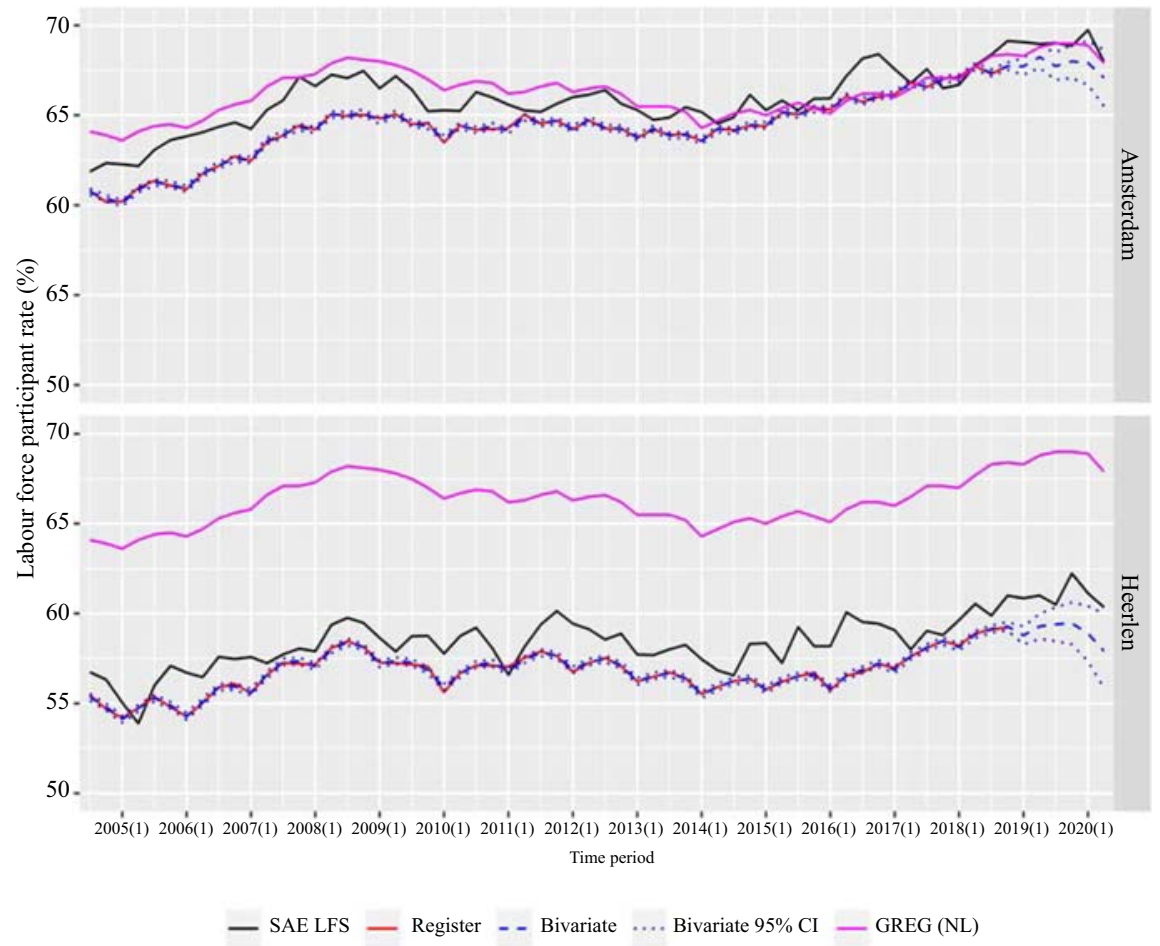

Fig. 10. Nowcasting the register series in the COVID-19 pandemic for the municipalities Amsterdam and Heerlen (using the GREG LFS estimates at national level as auxiliary series).

area prediction and the register series observed up to the fourth quarter of 2018. For reference, the LFS national GREG estimates are also included.

If the LFS GREG estimates at the national level are used as an auxiliary series, a correlation between the slope disturbance terms of 0.9 is found for Amsterdam and Heerlen. If the municipal small area predictions are used as an auxiliary series, the correlation between the slope disturbance terms is 0.9 again for Amsterdam and 1.0 for Heerlen. The LFS GREG estimates at the national level as well as the municipal small area predictions for Heerlen and Amsterdam show a clear drop for the employment rate in the second quarter of 2020. This drop is clearly picked up by the nowcasts for Amsterdam and Heerlen if the LFS GREG estimates at the national level are used as an auxiliary series. If the LFS municipal small area prediction are used as an auxiliary series, then the nowcast for Amsterdam still picks up a clear, but less pronounced, drop for the second quarter of 2020. The nowcast for Heerlen does not show a drop for the last quarter, despite the correlation between the slope disturbance terms being larger if the municipal small area prediction are used as an auxiliary series. The reason behind this observation is that the standard errors of the LFS GREG estimates at the national level are clearly smaller compared to the MSEs of the municipal small area predictions. Using an auxiliary series at a high aggregation level, however, might result in larger bias in the nowcasts. At the moment of doing this nowcast exercise, it is not possible to evaluate the nowcasts, since the final values of the register series are not available. 


\section{Discussion}

An advantage of using registers for the production of official statistics rather than sample surveys is that they allow for the compilation of very accurate statistics at very detailed breakdowns, since it approximates a complete enumeration of the finite target population. In addition, data collection costs are small compared to sample surveys and do not contribute to response burden. Examples in the Netherlands in which reliable information for statistical purposes is obtained from administrative data are income statistics, statistics on poverty rates, short term business statistics and statistics on the labour force participation, since they are all derived from tax administrations and administrations on social benefits. On the other hand, these data sources are not always timely. This applies, in particular, to tax administrations on income data.

In this article, a nowcast method is proposed to improve the timeliness of very detailed regional statistics on the labour force participation rate derived from a tax register, by using more timely survey estimates obtained from the labour force survey (LFS). The LFS data for a particular reference year become available eight months in advance of the preliminary register statistics, and one year and eight months in advance of the final register statistics. As a first step, estimates for quarterly municipal figures on labour force participation rates are constructed using the LFS data with a small area estimation technique that is based on a cross-sectional small area estimation method. In this application, the unit level model of Battese et al. (1988) is used. This gives rise to a time series of timely quarterly small area estimates at the municipal level. These series are combined with the more precise, but less timely, quarterly series on labour force participation rates derived from the tax register in a bivariate structural time series model. The model uses the correlation between the disturbance terms of the stochastic trend component of the labour force participation rates in the survey and register sources. This idea was introduced by Harvey and Chung (2000) the other way around, that is, to improve UK LFS estimates with claimant counts as an auxiliary series. Van den Brakel et al. (2017) used a similar state space model to improve the timeliness of the Dutch consumer confidence survey with a related more timely series derived from social media platforms. The model proposed in this article accounts for the uncertainty in the auxiliary series by modelling the sampling error in the small area estimates of the LFS as well as the autocorrelation in the sampling error due to the panel overlap.

The proposed methods are applied to estimate labour force participation rates at both the municipal and neighbourhood level using the register data with a one year lag as the main series and the LFS small area estimates as the auxiliary series. The introduction of an auxiliary survey series at the municipal level decreases the standard errors of the predicted measurements of the one-year lag (nowcasts) on average by $20 \%$, both at the municipal and at the neighbourhood level. The gains in terms of RRMSEs are smaller. The reduction in the variance of the predicted estimates hinges on two contributing factors: (1) the strength of the correlation between the register and survey series and (2) the amount of uncertainty in the small area estimates of the auxiliary series.

By choosing a series that correlates well with the main series and has a small variance component, for example, LFS estimates for labour force participation rates at the national level, the reduction in the standard errors in the bivariate analysis may be as large as $40 \%$. 
On the other hand, using a noisy auxiliary series in the bivariate analysis does not lead to large improvements in the accuracy of the predicted estimates. For smaller municipalities, replacing the municipal auxiliary LFS series of the labour force participation rate by a series at a higher regional level (province or even at the national level) may therefore be a better choice.

Predictions of labour force participation rates from the time series modelling approach at the regional level (municipalities and neighbourhoods) were compared with the actual, final measurements. Predictions root relative mean squares measures were calculated for both the univariate and bivariate modelling results. These measures show that in the case of a noisy (municipal) auxiliary series on labour force participation rates, the bivariate time series model does not produce more accurate nowcasts than the univariate model. When using a less volatile auxiliary series on labour force participation at a higher regional level (the national level), the bivariate model produces more accurate results than the univariate model.

The proposed method is extended to the start of the COVID-19 pandemic. It is found that the extent to which the nowcasts pick up the turning point induced by the lockdown of the Corona crisis depends on the accuracy of the auxiliary series. If the series of LFS GREG estimates at the national level with small standard errors are used, then the nowcasts pick up the turning point induced by the lockdown. If municipal small area predictions are used, then the turning point in the nowcasts is less pronounced because these auxiliary series have larger MSEs.

In the approach discussed in this article, the explanatory variable is observed with sampling error. From this point of view, the approach followed in this article has some similarities with the area level models used in small area estimation, where the auxiliary variables are observed with measurement error (Ybarra and Lohr 2008). In particular, the bivariate structural time series model in this article can be seen as a temporal version of the structural measurement error model, as discussed in Bell et al. (2019).

The method introduced in this article may be used for other applications where lagged register statistics can be nowcasted by using correlated auxiliary series observed from a more timely sample survey. One research line is to develop multivariate structural time series models in which the register series for all neighbourhoods within a municipality are combined with the LFS series at a higher regional level. Such models can also account for and profit from the correlation between the register series of the neighbourhoods. Highdimensionality problems will likely occur but can be handled with a dynamic factor model in state space form, where common factors are derived in a first step using principal components (see e.g., Giannone et al. 2008). Another research direction would be to extend the small area estimation approach for LFS figures to smaller regional domains using temporal and cross-sectional correlations. Finally, the (bivariate) time series model can also be applied to improve the accuracy of the LFS series as well, even if this series contains more timely data. Van den Brakel and Krieg (2016) showed that monthly estimates for the unemployed labour force derived from LFS data can be improved with claimant count series, even if the last two months of claimant counts are not available. Analyses on municipal labour force participation rates suggest that a univariate time series modelling approach can reduce the standard errors of the survey series small area estimates. Bivariate analyses with lagged register data seem to lead to further small improvements. 


\section{Appendix}

\subsection{Explanation Variables}

Employed Reg: employed according to register (Polis-register);

0: not employed

1: employed

Sex:

1: men

2: women

Age (5): age in five classes:

1: $15-24$

2: $25-34$

3: $35-44$

4: $45-54$

5: $55-75$

Age (3): age in three classes:

1: $15-24$

2: $25-44$

3: $45-75$

Ethnicity (3): migration background in three classes;

1: Dutch background

2: Western background ${ }^{1}$

3: Non-western background ${ }^{2}$

Ethnicity (7): migration background in seven classes;

1: Dutch background

2: Moroccan

3: Turkish

4: Surinam

5: Antillen/Aruba

6: Other non-Western ${ }^{2}$

7: Western background ${ }^{1}$

Employment Office:

1: Not registered at the Employment Office

2: Registered at the employment office with a job and searching for work

3: Registered as unemployed less than one year

4: Registered as unemployed for one to four years

5: Registered as unemployed for more than four years

Border municipality (2) municipality is close to the border:

1: Not a border municipality

2: Is a border municipality

HH Type: Household type in three classes:

1: One person household

2: Household with children

3: Other household types 
WaveNr.: wave number in the panel $(1, \ldots, 5)$

${ }^{1}$ Western immigrant are defined as: all European countries excluding Turkey, North America, Canada, Australia, New-Zealand, Japan and Indonesia.

${ }^{2}$ Non-Western immigrant are defined as: Turkey, African countries, Latin America, all Asian countries with the exception of Japan and Indonesia.

\subsection{Model Diagnostics (2003-2014 Series)}

Meaning of table columns:

Value $=$ value of test statistic

$\mathrm{L}=$ lower bound of $95 \%$-confidence interval (n.a. = not applicable)

$\mathrm{U}=$ upper bound of $95 \%$-confidence interval

Table 8. Univariate model register.

\begin{tabular}{|c|c|c|c|c|c|c|c|c|c|}
\hline \multirow[t]{2}{*}{ Diagnostic } & \multicolumn{3}{|c|}{ Amsterdam } & \multicolumn{3}{|c|}{ Heerlen } & \multicolumn{3}{|l|}{ Haren } \\
\hline & Value & $\mathrm{L}$ & $\mathrm{U}$ & Value & $\mathrm{L}$ & $\mathrm{U}$ & Value & $\mathrm{L}$ & $\mathrm{U}$ \\
\hline Mean & 0.089 & & & 0.051 & & & 0.039 & & \\
\hline Variance & 0.991 & & & 0.997 & & & 0.998 & & \\
\hline Skewness & 0.689 & & & 0.353 & & & 0.558 & & \\
\hline Kurtosis & 4.172 & & & 2.894 & & & 3.590 & & \\
\hline $\begin{array}{l}\text { Bowman- } \\
\text { Shenton }^{1}\end{array}$ & 5.870 & & 5.991 & 0.916 & & 5.991 & 2.861 & & 5.991 \\
\hline $\begin{array}{l}\text { Ljung- } \\
\text { Box }^{2}\end{array}$ & 4.278 & & 21.02 & 8.953 & & 21.02 & 10.16 & & 21.02 \\
\hline $\begin{array}{l}\text { Durbin- } \\
\text { Watson }^{3}\end{array}$ & 1.979 & 1.402 & 2.597 & 1.932 & 1.402 & 2.597 & 1.807 & 1.402 & 2.597 \\
\hline $\begin{array}{l}\text { F-test } \\
\text { heterosce- } \\
\text { dasticity }^{4}\end{array}$ & $11.40^{*}$ & 0.305 & 3.277 & 2.201 & 0.305 & 3.277 & 1.768 & 0.305 & 3.277 \\
\hline
\end{tabular}

*test statistic outside $95 \%$ confidence interval under null hypothesis.

Table 9. Bivariate model register innovations.

\begin{tabular}{|c|c|c|c|c|c|c|c|c|c|}
\hline \multirow[t]{2}{*}{ Diagnostic } & \multicolumn{3}{|c|}{ Amsterdam } & \multicolumn{3}{|c|}{ Heerlen } & \multicolumn{3}{|c|}{ Haren } \\
\hline & Value & $\mathrm{L}$ & $\mathrm{U}$ & Value & $\mathrm{L}$ & $\mathrm{U}$ & Value & $\mathrm{L}$ & $\mathrm{U}$ \\
\hline Mean & 0.107 & & & 0.043 & & & 0.094 & & \\
\hline Variance & 0.976 & & & 0.991 & & & 0.915 & & \\
\hline Skewness & 0.683 & & & 0.417 & & & 0.608 & & \\
\hline Kurtosis & 3.927 & & & 3.044 & & & 3.832 & & \\
\hline $\begin{array}{l}\text { Bowman- } \\
\text { Shenton }^{1}\end{array}$ & 4.766 & & 5.991 & 1.224 & & 5.991 & 3.804 & & 5.991 \\
\hline $\begin{array}{l}\text { Ljung- } \\
\text { Box }^{2}\end{array}$ & 4.526 & & 21.02 & 10.39 & & 21.02 & 9.010 & & 21.02 \\
\hline $\begin{array}{l}\text { Durbin- } \\
\text { Watson }^{3}\end{array}$ & 1.988 & 1.395 & 2.604 & 1.901 & 1.395 & 2.604 & 1.858 & 1.395 & 2.604 \\
\hline $\begin{array}{l}\text { F-test } \\
\text { heterosce- } \\
\text { dasticity }^{4}\end{array}$ & $7.151^{*}$ & 0.305 & 3.277 & 1.336 & 0.305 & 3.277 & 1.748 & 0.305 & 3.277 \\
\hline
\end{tabular}

*test statistic outside $95 \%$ confidence interval under null hypothesis. 
Table 10. Bivariate model LFS innovations.

\begin{tabular}{|c|c|c|c|c|c|c|c|c|c|}
\hline \multirow[t]{2}{*}{ Diagnostic } & \multicolumn{3}{|c|}{ Amsterdam } & \multicolumn{3}{|c|}{ Heerlen } & \multicolumn{3}{|l|}{ Haren } \\
\hline & Value & $\mathrm{L}$ & $\mathrm{U}$ & Value & $\mathrm{L}$ & $\mathrm{U}$ & Value & $\mathrm{L}$ & $\mathrm{U}$ \\
\hline Mean & -0.117 & & & -0.100 & & & -0.083 & & \\
\hline Variance & 1.028 & & & 0.951 & & & 0.933 & & \\
\hline Skewness & 0.406 & & & 0.070 & & & 0.068 & & \\
\hline Kurtosis & 2.644 & & & 2.953 & & & 2.108 & & \\
\hline $\begin{array}{l}\text { Bowman- } \\
\text { Shenton }^{1}\end{array}$ & 1.378 & & 5.991 & 0.038 & & 5.991 & 1.424 & & 5.991 \\
\hline $\begin{array}{l}\text { Ljung- } \\
\text { Box }^{2}\end{array}$ & 13.05 & & 21.02 & 12.63 & & 21.02 & 13.76 & & 21.02 \\
\hline $\begin{array}{l}\text { Durbin- } \\
\text { Watson }\end{array}$ & 1.880 & 1.395 & 2.604 & 1.688 & 1.395 & 2.604 & 1.569 & 1.395 & 2.604 \\
\hline $\begin{array}{l}\text { F-test } \\
\text { heterosce- } \\
\text { dasticity }\end{array}$ & 1.148 & 0.305 & 3.277 & 2.363 & 0.305 & 3.277 & 1.092 & 0.305 & 3.277 \\
\hline
\end{tabular}

Table 11. Autocorrelations and cross correlation of standardised innovations (bivariate model).

\begin{tabular}{llll}
\hline Type of correlation & Amsterdam & Heerlen & Haren \\
\hline Autocorrelation (register innovations) & & & \\
$\quad$ Lag 1 & 0.000 & 0.016 & 0.043 \\
Lag 2 & -0.038 & 0.050 & -0.223 \\
Lag 3 & 0.130 & 0.110 & 0.013 \\
Cross correlation (register-LFS innovations) & 0.166 & 0.075 & 0.132 \\
\hline
\end{tabular}

Table 12. Bivariate model register innovations (multiplicative model).

\begin{tabular}{|c|c|c|c|}
\hline \multirow[t]{2}{*}{ Diagnostic } & \multicolumn{3}{|c|}{ Heerlen } \\
\hline & Value & $\mathrm{L}$ & $\mathrm{U}$ \\
\hline Mean & 0.043 & & \\
\hline Variance & 0.989 & & \\
\hline Skewness & 0.398 & & \\
\hline Kurtosis & 3.041 & & \\
\hline Bowman-Shenton $^{1}$ & 1.112 & & 5.991 \\
\hline Ljung-Box ${ }^{2}$ & 10.11 & & 21.02 \\
\hline Durbin-Watson ${ }^{3}$ & 1.890 & 1.395 & 2.604 \\
\hline F-test heteroscedasticity ${ }^{4}$ & 1.438 & 0.305 & 3.277 \\
\hline
\end{tabular}


Table 13. Bivariate model LFS innovations (multiplicative model).

\begin{tabular}{llll}
\hline Diagnostic & \multicolumn{3}{l}{ Heerlen } \\
& Value & L & U \\
\hline Mean & -0.100 & & \\
Variance & 0.951 & & \\
Skewness & 0.058 & & \\
Kurtosis & 3.080 & & \\
Bowman-Shenton $^{1}$ & 0.035 & & 5.991 \\
Ljung-Box $^{2}$ & 11.94 & & 21.02 \\
Durbin-Watson $^{3}$ & 1.707 & 1.395 & 2.604 \\
F-test heteroscedasticity $^{4}$ & 2.438 & 0.305 & 3.277 \\
\hline
\end{tabular}

${ }^{1}$ Bowman-Shenton test on normality of the standardised innovations: distribution under $\mathrm{H}_{0}: \chi_{2}^{2}$

${ }^{2}$ Liung-Box test on autocorrelation of the first eight lags in the standardised innovations: distribution under $\mathrm{H}_{0}: \chi_{7}^{2}$

${ }^{3}$ Durbin-Watson test on autocorrelation in the standardised residuals: distribution under $\mathrm{H}_{0}$ approximated with $N\left(2, \frac{4}{\mathrm{~T}}\right)$

${ }^{4}$ F-test for heteroscedasticity in the standardised innovations: distribution under $\mathrm{H}_{0}: \chi_{11}^{11}$

\section{References}

Arima, S., W.R. Bell, G.S. Datta, C. Franco, and B. Liseo. 2017. "Multivariate Fay-Herriot Bayesian estimation of small area means under functional measurement error." Journal of the Royal Statistical Society Series A, 180: 1191-1209. DOI: https://cran.rproject.org/web/packages/hbsae/hbsae.pdf.

Bakker, B.F.M. 2012. "Estimating the Validity of Administrative Variables." Statistica Neerlandica, 66: 8-17. DOI: https://doi.org/10.1111/j.1467-9574.2011.00504.x.

Battese, G.E., R.M. Harter, and W.A. Fuller. 1988. "An error components model for prediction of county crop areas using survey and satellite data." Journal of the American Statistical Association. 83: 28-36. DOI: https://doi.org/10.2307/2288915.

Bell, W.R., H.C. Chung, G.S. Datta, and C. Franco. 2019. "Measurement error in small area estimation: Functional versus structural versus naïve models." Survey Methodology 45: 61-80. Available at: https://www150.statcan.gc.ca/n1/pub/12-001-x/2019001/ article/00005-eng.htm (accessed August 2020).

Bijlsma, I., J.A. van den Brakel, R. van der Velden, and J. Allen. 2020. "Estimating literacy levels at a detailed regional level; An application using Dutch data." Journal of Official Statistics, 36: 251-274. DOI: http://dx.doi.org/10.2478/JOS-2020-0014.

Blight, B.J.N., and A.J. Scott. 1973. "A stochastic model for repeated surveys." Journal of the Royal Statistical Society Series B, 35: 61-66. DOI: https://doi.org/10.1111/j.25176161.1973.tb00936.x.

Bollineni-Balabay, O., J.A. van den Brakel, and F. Palm. 2017. "State space time series modelling of the Dutch Labour Force Survey: Model selection and mean squared error estimation." Survey Methodology, 43: 41-67. Available at: http://www.statcan.gc.ca/ pub/12-001-x/2017001/article/14819-eng.htm (accessed November 2019). 
Boonstra, H.J. 2012. hbsae: Hierarchical Bayesian Small Area Estimation.R package version 1.0. https://cran.r-project.org/web/packages/hbsae/hbsae.pdf (accessed February 2020).

Boonstra, H.J. and J.A. van den Brakel. 2019. "Estimation of level and change for unemployment using structural time series models." Survey Methodology, 45: 395-425. Available at: http://www.statcan.gc.ca/pub/12-001-x/2019003/article/ 00005-eng.htm (accessed February 2020).

Boonstra, H.J., J.A. van den Brakel, B. Buelens, S. Krieg, and M. Smeets. 2008. "Towards small area estimation at Statistics Netherlands." Metron, LXVI (1): 21-49.

Boonstra, H.J., B. Buelens, and M. Smeets. 2007. Estimation of municipal unemployment fractions - a simulation study comparing different small area estimators. Technical report, BPA-no. DMH-2007-04-20-HBTA, Statistics Netherlands, Heerlen. Available at: https://www.google.com/url?sa=t\&rct=j\&q=\&esrc=s\&source=web\&cd=\&cad=rja \&uact=8\&ved=2ahUKEwjgldbMivrzAhVKDuwKHW-jCQEQFnoECAoQAQ\&url= https $\% 3 \mathrm{~A} \% 2 \mathrm{~F} \% 2 \mathrm{Fwww} . c b s . n 1 \% 2 \mathrm{~F}-\% 2 \mathrm{Fmedia} \% 2 \mathrm{Fimported} \% 2 \mathrm{Fdocuments} \% 2 \mathrm{~F} 2011$ \%2F02\%2F2011-x10-02.pdf\%3Fla\%3Dnl-nl\&usg=AOvVaw3n58-noqm4X0g4z5v28 QSL (accessed June 2020).

Boonstra, H.J., B. Buelens, K. Leufkens, and M. Smeets. 2011. Small area estimates of labour status in Dutch municipalities. Technical Report 201102, Statistics Netherlands. CBS Statline (2017). Statline statistical database: Avaliable at: https://opendata.cbs.nl/ statline/\#/CBS/nl/dataset/83524NEDtable?dl = 40C7/ (accessed November 2021).

Choi, H., and H. Varian. 2012. "Predicting the Present with Google Trends." Economic Record 88 Supplement s1: 2-9. DOI: https://doi.org/10.1111/j.1475-4932.2012. 00809.x.

Cochran, W. 1977. Sampling Theory. New York: John Wiley \& Sons, Inc.

Daas, P., and M. Puts. 2014. "Big data as a source of statistical information." The Survey Statistician, 69: 22-31. Available at: http://isi-iass.org/home/wp-content/uploads/n692014-01-issn.pdf (accessed November 2019).

Datta, G.S., and M. Ghosh. 1991. "Bayesian Prediction in Linear Models: Applications to Small Area Estimation.” The Annals of Statistics, 19(4): 1748-1770. DOI: https://doi. org/10.1214/aos/1176348369.

Datta, G., P. Lahiri, T. Maiti, and K. Lu. 1999. "Hierarchical Bayes estimation of unemployment rates for the states of the U.S." Journal of the American Statistical Association 94: 1074-1082. DOI: https://doi.org/10.2307/2669921.

Doornik, J.A. 2009. An Object-oriented Matrix Programming Language Ox 6. London: Timberlake Consultants Press.

Durbin, J., and S.J. Koopman. 2012. Time Series Analysis by State Space Methods (2nd edition). Oxford: Oxford University Press.

Fay, R.E., and R.A. Herriot. 1979. "Estimates of income for small places: an application of James-Stein procedures to Census data." Journal of the American Statistical Association 74: 269-277. DOI: https://doi.org/10.1080/01621459.1979.10482505.

Giannone, D., L. Reichlin, and D. Small. 2008. "Nowcasting: The real-time informational content of macroeconomic data." Journal of Monetary Economics 55: 665-676. DOI: https://doi.org/10.1016/j.jmoneco.2008.05.010. 
Hand, D. 2018. "Statistical challenges of administrative and transaction data." Journal of the Royal Statistical Society series A, 181: 1-51. DOI: https://doi.org/10.1111/rssa. 12315.

Harvey, A.C. 1989. Forecasting, Structural Time Series Models and the Kalman Filter. Cambridge: Cambridge University Press.

Harvey, A.C., and C.H. Chung. 2000. "Estimating the underlying change in unemployment in the UK." Journal of the Royal Statistical Society Series A, 163: 303-339. DOI: https://doi.org/10.1111/1467-985X.00171.

Hobza, T., and D. Morales. 2016. "Empirical best prediction under unit-level logit mixed models." Journal of Official Statistics, 32: 661-692. DOI: https://doi.org/10.1515/jos2016-0034.

Hobza, T., D. Morales, and L. Santamaria. 2018. "Small area estimation of poverty proportions under unit-level temporal binomial-logit mixed models." TEST, 27: 270-294. DOI: https://doi.org/10.1007/s11749-017-0545-3.

Koopman, S.J., A.C. Harvey, J.A. Doornik, and N. Shephard. 2007. STAMP8: Structural Time Series Analyser, Modeller and Predictor. London: Timberlake.

Koopman, S.J., N. Shephard, and J.A. Doornik. 2008. SsfPack 3.0: Statistical Algorithms for Models in State Space Form, London: Timberlake Consultants Press.

MacGibbon, B., and T.J. Tomberlin. 1989. "Small Area Estimates of Proportions via Empirical Bayes Techniques.” Survey Methodology, 15: 237-252. Available at: https:// www150.statcan.gc.ca/n1/pub/12-001-x/1989002/article/14565-eng.pdf (accessed August 2021).

Malec, D., J. Sedransk, C.L. Moriarity, and F.B. Leclere. 1997. "Small Area Inference for Binary Variables in the National Health Interview Survey." Journal of the American Statistical Association, 92: 815-826. DOI: https://doi.org/10.1080/01621459.1997. 10474037.

Marino, M.F., M.G. Ranalli, N. Salvati, and M. Alfo. 2019. "Semiparametric empirical best prediction for small area estimation of unemployment indicators." The Annals of Applied Statistics, 13: 1166-1197. DOI: https://doi.org/10.1214/18-AOAS1226.

Pfeffermann, D. 2002. "Small area estimation - new developments and directions." International Statistical Review 70: 125-143. DOI: https://doi.org/10.1111/j.17515823.2002.tb00352.x.

Pfeffermann, D. 2013. "New important developments in small area estimation." Statistical Science 28: 40-68. DOI: https://doi.org/10.1214/12-STS395.

Pfeffermann, D., and L. Burck. 1990. "Robust Small Area Estimation combining Time Series and Cross-sectional Data." Survey Methodology, 16: 217-237. Available at: https://www150.statcan.gc.ca/n1/en/pub/12-001-x/1990002/article/14534eng.pdf?st $=$ pRQklC03 (accessed October 2019).

Pfeffermann, D., J.L. Eltinge, and L.D. Brown. 2015. "Methodological issues and challenges in the production of official statistics." Journal of Survey Statistics and Methodology 3: 425-483. DOI: https://doi.org/10.1093/jssam/smv035.

Pfeffermann, D., and R. Tiller. 2005. "Bootstrap approximation to prediction MSE for state-space models with estimated parameters." Journal of Time Series Analysis, 26: 893-916. DOI: https://doi.org/10.1111/j.1467-9892.2005.00448.x. 
Pfeffermann, D., and R. Tiller. 2006. "Small area estimation with state space models subject to benchmark constraints." Journal of the American Statistical Association 101: 1387-1397. DOI: https://doi.org/10.1198/016214506000000591.

Rao, J.N.K., and I. Molina. 2015. Small Area Estimation, second edition. New York: John Wiley \& Sons.

Rao, J.N.K., and M. Yu. 1994. "Small area estimation by combining time-series and crosssectional data." The Canadian Journal of Statistics 22: 511-528. DOI: https://doi. org/10.2307/3315407.

Särndal, C.-E., B. Swensson, and J. Wretman. 1992. Model Assisted Survey Sampling. New-York: Springer-Verlag.

Van den Brakel, J.A., E. Söhler, P. Daas, and B. Buelens. 2017. "Social media as a data source for official statistics; the Dutch Consumer Confidence Index." Survey Methodology 43: 183-210. Available at: http://www.statcan.gc.ca/pub/12-001-x/ 2017002/article/54871-eng.htm (accessed October 2019).

Van den Brakel, J.A., and S. Krieg. 2015. "Dealing with small sample sizes, rotation group bias and discontinuities in a rotating panel design." Survey Methodology 41: 267-296. Available at http://www.statcan.gc.ca/pub/12-001-x/2015002/article/14231- eng.pdf (accessed November 2019).

Van den Brakel, J.A., and S. Krieg. 2016. "Small area estimation with state-space common factor models for rotating panels." Journal of the Royal Statistical Society Series A. 179: 763-791. DOI: https://doi.org/10.1111/rssa.12158.

Vosen, M., and T. Schmidt. 2011. "Forecasting private consumption: survey-based indicators vs. Google trends." Journal of Forecasting 30: 565-578. DOI: https://doi. org/10.1002/for.1213.

Wallgren, A., and B. Wallgren. 2007. Register-based statistics; Administrative data for statistical purposes. John Wiley \& Sons, West Sussex.

Ybarra, L.M.R., and S.L. Lohr. 2008. "Small area estimation when auxiliary information is measured with error." Biometrika 95: 919-931. DOI: https://doi.org/10.1093/biomet/asn048.

You, Y., J. Rao, and J. Gambino. 2003. "Model-based unemployment rate estimation for the Canadian Labour Force Survey: A hierarchical Bayes approach." Survey Methodology 29: 25-32. Available at: https://www150.statcan.gc.ca/n1/pub/12-001x/2003001/article/6602-eng.pdf (accessed November 2019).

Received March 2020

Revised September 2020

Accepted April 2021 